\title{
More on Generalizations and Modifications of Iterative Methods for Solving Large Sparse Indefinite Linear Systems
}

\author{
Jen-Yuan Chen, ${ }^{1}$ David R. Kincaid, ${ }^{2}$ and Yu-Chien $\mathrm{Li}^{1}$ \\ ${ }^{1}$ Department of Mathematics, National Kaohsiung Normal University, Kaohsiung 81201, Taiwan \\ ${ }^{2}$ Institute for Computational Engineering and Sciences, The University of Texas at Austin, Austin, TX 78712, USA
}

Correspondence should be addressed to Jen-Yuan Chen; jchen@nknu.edu.tw

Received 22 May 2014; Accepted 17 July 2014; Published 27 October 2014

Academic Editor: Giuseppe Marino

Copyright (C) 2014 Jen-Yuan Chen et al. This is an open access article distributed under the Creative Commons Attribution License, which permits unrestricted use, distribution, and reproduction in any medium, provided the original work is properly cited.

Continuing from the works of $\mathrm{Li}$ et al. (2014), $\mathrm{Li}$ (2007), and Kincaid et al. (2000), we present more generalizations and modifications of iterative methods for solving large sparse symmetric and nonsymmetric indefinite systems of linear equations. We discuss a variety of iterative methods such as GMRES, MGMRES, MINRES, LQ-MINRES, QR MINRES, MMINRES, MGRES, and others.

\section{Introduction}

When solving large sparse linear systems of the form

$$
\mathbf{A x}=\mathbf{b},
$$

in which the coefficient matrix $\mathbf{A}$ is indefinite, there are basis methods and a variety of generalizations and modifications of them. For example, basic iterative methods for symmetric indefinite linear systems are the MINRES method and the SYMMLQ method, while a basic method for nonsymmetric linear systems is the GMRES method. (See, e.g., Lanczos [1], Golub and Van Loan [2], Paige and Saunders [3], Saad [4], and Saad and Schultz [5].)

In Section 2, we review the Arnoldi process and present background material. In Sections 3 and 4, we describe the LQ-MINRES and the QR-MINRES methods, respectively, as well as discussing their relationship in Section 5. In Section 6, we take a closer look at the QR-MINRES method and the SYMMQR method. In Sections 7 and 8, we describe the modified MINRES (MMINRES) method and the generalized QRMINRES method, respectively. In Section 9, we review the GMRES method. Finally, we discuss the differences between the modified MINRES (MMINRES) method and the modified GMRES (MGMRES) method, in Section 10.

\section{Arnoldi Process}

First, we assume that matrix $\mathbf{A}$ is symmetric. In $[6,7]$, we use a short term recurrence to generate orthonormal vectors $\left\{\mathbf{w}^{(0)}, \mathbf{w}^{(1)}, \mathbf{w}^{(2)}, \ldots, \mathbf{w}^{(n-3)}, \mathbf{w}^{(n-2)}, \mathbf{w}^{(n-1)}\right\}$ as follows:

$$
\begin{aligned}
\mathbf{u}^{(0)} & =\operatorname{arbitrary}, \\
\mathbf{r}^{(0)} & =\mathbf{b}-\mathbf{A} \mathbf{u}^{(0)}, \\
\widetilde{\mathbf{w}}^{(0)} & =\mathbf{r}^{(0)}, \\
\sigma_{0}= & \sqrt{\left\langle\widetilde{\mathbf{w}}^{(0)}, \widetilde{\mathbf{w}}^{(0)}\right\rangle,} \\
\mathbf{w}^{(0)}= & \left(\frac{1}{\sigma_{0}}\right) \widetilde{\mathbf{w}}^{(0)}, \\
\text { for } \quad & i=1,2, \ldots, n-1, \\
& \alpha_{i-1}=\left\langle\mathbf{A} \mathbf{w}^{(i-1)}, \mathbf{w}^{(i-1)}\right\rangle, \\
& \beta_{i-1}=\left\langle\mathbf{A} \mathbf{w}^{(i-1)}, \mathbf{w}^{(i-2)}\right\rangle, \\
& \widetilde{\mathbf{w}}^{(i)}=\mathbf{A w}^{(i-1)}-\alpha_{i-1} \mathbf{w}^{(i-1)}-\beta_{i-1} \mathbf{w}^{(i-2)},
\end{aligned}
$$




$$
\begin{aligned}
& \sigma_{i}=\sqrt{\left\langle\widetilde{\mathbf{w}}^{(i)}, \widetilde{\mathbf{w}}^{(i)}\right\rangle,} \\
& \mathbf{w}^{(i)}=\left(\frac{1}{\sigma_{i}}\right) \widetilde{\mathbf{w}}^{(i)},
\end{aligned}
$$

end for.

Here we assume that $\mathbf{w}^{(-1)}=\mathbf{0}$ and $\sigma_{i} \neq 0$, for all $i$. If we let $\widetilde{\mathbf{w}}^{(0)}=\mathbf{r}^{(0)}=\mathbf{b}-\mathbf{A} \mathbf{u}^{(0)}$, then the subspace,

$$
\operatorname{Span}\left\{\mathbf{w}^{(0)}, \mathbf{w}^{(1)}, \mathbf{w}^{(2)}, \ldots, \mathbf{w}^{(n-3)}, \mathbf{w}^{(n-2)}, \mathbf{w}^{(n-1)}\right\},
$$

is equivalent to the Krylov subspace

$$
\begin{gathered}
\mathscr{K}_{n}\left(\mathbf{r}^{(0)}, \mathbf{A}\right)=\operatorname{Span}\left\{\mathbf{r}^{(0)}, \mathbf{A} \mathbf{r}^{(0)}, \mathbf{A}^{2} \mathbf{r}^{(0)}, \mathbf{A}^{3} \mathbf{r}^{(0)}, \ldots, \mathbf{A}^{n-3} \mathbf{r}^{(0)}\right. \\
\left.\mathbf{A}^{n-2} \mathbf{r}^{(0)}, \mathbf{A}^{n-1} r^{(0)}\right\}
\end{gathered}
$$

Consequently, we have the following properties, for $(0 \leq i$, $j \leq n-1)$ :

$$
\begin{aligned}
\left\langle\mathbf{w}^{(i)}, \mathbf{w}^{(j)}\right\rangle & =\delta_{i j}, \\
\beta_{i} & =\sigma_{i},
\end{aligned}
$$

as well as these matrix equations

$$
\begin{aligned}
\mathbf{A W}_{n-1} & =\mathbf{W}_{n-1} \mathbf{T}_{n}+\sigma_{n} \mathbf{w}^{(n)} \mathbf{e}_{n}^{T} \\
& =\mathbf{W}_{n} \widetilde{\mathbf{T}}_{n+1}, \\
& \mathbf{W}_{n-1}^{T} \mathbf{W}_{n-1}=\mathbf{I},
\end{aligned}
$$

where

$$
\begin{aligned}
\mathbf{W}_{n-1}= & {\left[\mathbf{w}^{(0)}, \mathbf{w}^{(1)}, \mathbf{w}^{(1)}, \ldots, \mathbf{w}^{(n-3)}, \mathbf{w}^{(n-2)}, \mathbf{w}^{(n-1)}\right]_{n \times n} } \\
\mathbf{e}_{n}^{T}= & {[0,0,0, \ldots, 0,0,0,1]_{1 \times n}, } \\
\mathbf{T}_{n}= & {\left[\begin{array}{lllllll}
\alpha_{0} & \beta_{1} & & & & \\
\sigma_{1} & \alpha_{1} & \beta_{2} & & & & \\
& \sigma_{2} & \alpha & \alpha_{3} & & & \\
& & \ddots & \ddots & \ddots & & \\
& & & \sigma_{n-3} & \alpha_{n-3} & \beta_{n-2} & \\
& & & \sigma_{n-2} & \alpha_{n-2} & \beta_{n-1} \\
& & & & \sigma_{n-1} & \alpha_{n-1}
\end{array}\right]_{n \times n} }
\end{aligned}
$$$$
\widetilde{\mathbf{T}}_{n+1}=\left[\begin{array}{ccccccc}
\alpha_{0} & \beta_{1} & & & & & \\
\sigma_{1} & \alpha_{1} & \beta_{2} & & & & \\
& \sigma_{2} & \alpha_{2} & \beta_{3} & & & \\
& & \ddots & \ddots & \ddots & & \\
& & & \sigma_{n-3} & \alpha_{n-3} & \beta_{n-2} & \\
& & & & \sigma_{n-2} & \alpha_{n-2} & \beta_{n-1} \\
& & & & & \sigma_{n-1} & \alpha_{n-1} \\
& & & & & & \sigma_{n}
\end{array}\right]_{(n+1) \times n}
$$

$$
=\mathbf{T}_{n}+\mathbf{T}_{n} e_{n+1}^{T}
$$

Example. We illustrate (6), for the case $n=3$.

From (2) and (7), we have

$$
\begin{aligned}
& \sigma_{1} \mathbf{w}^{(1)}=\widetilde{\mathbf{w}}^{(1)}=\mathbf{A} \mathbf{w}^{(0)}-\alpha_{0} \mathbf{w}^{(0)}-\beta_{0} \mathbf{w}^{(-1)} \\
& \sigma_{2} \mathbf{w}^{(2)}=\widetilde{\mathbf{w}}^{(2)}=\mathbf{A} \mathbf{w}^{(1)}-\alpha_{1} \mathbf{w}^{(1)}-\beta_{1} \mathbf{w}^{(0)} \\
& \sigma_{3} \mathbf{w}^{(3)}=\widetilde{\mathbf{w}}^{(3)}=\mathbf{A} \mathbf{w}^{(2)}-\alpha_{2} \mathbf{w}^{(2)}-\beta_{2} \mathbf{w}^{(1)}
\end{aligned}
$$

Since $\mathbf{w}^{(-1)}=\mathbf{0}$, we obtain

$$
\begin{aligned}
& \mathbf{A} \mathbf{W}_{2}=\mathbf{A}\left[\begin{array}{lll}
\mathbf{w}^{(0)} & \mathbf{w}^{(1)} & \mathbf{w}^{(2)}
\end{array}\right]=\left[\begin{array}{lll}
\mathbf{A} \mathbf{w}^{(0)} & \mathbf{A} \mathbf{w}^{(1)} & \mathbf{A} \mathbf{w}^{(2)}
\end{array}\right] \\
& =\left[\begin{array}{c}
\beta_{0} \mathbf{w}^{(-1)}+\alpha_{0} \mathbf{w}^{(0)}+\sigma_{1} \mathbf{w}^{(1)} \\
\beta_{1} \mathbf{w}^{(0)}+\alpha_{1} \mathbf{w}^{(1)}+\sigma_{2} \mathbf{w}^{(2)} \\
\beta_{2} \mathbf{w}^{(1)}+\alpha_{2} \mathbf{w}^{(2)}+\sigma_{3} \mathbf{w}^{(3)}
\end{array}\right]^{T} \\
& =\left[\begin{array}{llll}
\mathbf{w}^{(0)} & \mathbf{w}^{(1)} & \mathbf{w}^{(2)} & \mathbf{w}^{(3)}
\end{array}\right]\left[\begin{array}{ccc}
\alpha_{0} & \beta_{1} & 0 \\
\sigma_{1} & \alpha_{1} & \beta_{2} \\
0 & \sigma_{2} & \alpha_{2} \\
0 & 0 & \sigma_{3}
\end{array}\right]_{4 \times 3} \\
& =\mathbf{W}_{3} \widetilde{\mathbf{T}}_{4}=\left[\begin{array}{ll}
\mathbf{W}_{2} & \mathbf{w}^{(3)}
\end{array}\right]\left[\begin{array}{ccc}
\mathbf{T}_{3} & \\
0 & 0 & \sigma_{3}
\end{array}\right] \\
& =\mathbf{W}_{2} \mathbf{T}_{3}+\sigma_{3} \mathbf{w}^{(3)} \mathbf{e}_{3}^{T} .
\end{aligned}
$$

So we obtain (6), with $n=3$ :

$$
\mathbf{A W}_{2}=\mathbf{W}_{2} \mathbf{T}_{3}+\sigma_{3} \mathbf{w}^{(3)} \mathbf{e}_{3}^{T}=\mathbf{W}_{3} \widetilde{\mathbf{T}}_{4}
$$

\section{LQ-MINRES Method}

We choose $\mathbf{u}^{(n)}$ such that $\mathbf{u}^{(n)}-\mathbf{u}^{(0)} \in \mathscr{K}_{n}\left(\mathbf{r}^{(0)}, \mathbf{A}\right)$. Hence, we have

$$
\begin{aligned}
& \mathbf{u}^{(n)}=\mathbf{u}^{(0)}+\mathbf{W}_{n-1} \mathbf{y}^{(n)}, \\
& \mathbf{r}^{(n)}=\mathbf{r}^{(0)}-\left(\mathbf{A W}_{n-1}\right) \mathbf{y}^{(n)} .
\end{aligned}
$$

For the MINRES method [3], we let

$$
\begin{aligned}
f\left(\mathbf{y}^{(n)}\right) & =\left(\mathbf{r}^{(n)}\right)^{T}\left(\mathbf{r}^{(n)}\right), \\
& =\left(\mathbf{r}^{(0)}-\mathbf{A} \mathbf{W}_{n-1} \mathbf{y}^{(n)}\right)^{T}\left(\mathbf{r}^{(0)}-\mathbf{A} \mathbf{W}_{n-1} \mathbf{y}^{(n)}\right) ;
\end{aligned}
$$

then

$$
\begin{aligned}
\nabla f\left(\mathbf{y}^{(n)}\right) & =-2 \mathbf{W}_{n-1}^{T} \mathbf{A}\left(\mathbf{r}^{(0)}-\mathbf{A} \mathbf{W}_{n-1} \mathbf{y}^{(n)}\right) \\
& =-2 \mathbf{W}_{n-1}^{T} \mathbf{A} \mathbf{r}^{(0)}+2 \mathbf{W}_{n-1}^{T} \mathbf{A}^{2} \mathbf{W}_{n-1} \mathbf{y}^{(n)}
\end{aligned}
$$

Letting $\nabla f\left(\mathbf{y}^{(n)}\right)=\mathbf{0}$, we can minimize $\left\|\mathbf{r}^{(n)}\right\|$ by solving this linear system for $\mathbf{y}^{(n)}$

$$
\left(\mathbf{W}_{n-1}^{T} \mathbf{A}^{2} \mathbf{W}_{n-1}\right) \mathbf{y}^{(n)}=\mathbf{W}_{n-1}^{T} \mathbf{A} \mathbf{r}^{(0)}
$$


First, using (6), (7), and (5), we expand the coefficient matrix on the left-hand side of linear system (18)

$$
\begin{aligned}
\mathbf{W}_{n-1}^{T} \mathbf{A}^{2} \mathbf{W}_{n-1} \\
=\left(\mathbf{W}_{n-1}^{T} \mathbf{A}\right)\left(\mathbf{A} \mathbf{W}_{n-1}\right) \\
=\left(\mathbf{A} \mathbf{W}_{n-1}\right)^{T}\left(\mathbf{A} \mathbf{W}_{n-1}\right) \\
=\left(\mathbf{W}_{n-1} \mathbf{T}_{n}+\sigma_{n} \mathbf{w}^{(n)} \mathbf{e}_{n}^{T}\right)^{T}\left(\mathbf{W}_{n-1} \mathbf{T}_{n}+\sigma_{n} \mathbf{w}^{(n)} \mathbf{e}_{n}^{T}\right) \\
=\left(\mathbf{T}_{n}^{T} \mathbf{W}_{n-1}^{T}+\sigma_{n} \mathbf{e}_{n}\left(\mathbf{w}^{(n)}\right)^{T}\right)\left(\mathbf{W}_{n-1} \mathbf{T}_{n}+\sigma_{n} \mathbf{w}^{(n)} \mathbf{e}_{n}^{T}\right) \\
=\mathbf{T}_{n}^{T} \mathbf{T}_{n}+\sigma_{n}^{2} \mathbf{e}_{n} \mathbf{e}_{n}^{T}=\mathbf{T}_{n}^{2}+\sigma_{n}^{2} \mathbf{e}_{n} \mathbf{e}_{n}^{T},
\end{aligned}
$$

since $\mathbf{T}_{n}$ is symmetric. Second, we exam the right-hand side vector in linear system (18)

$$
\left(\mathbf{W}_{n-1}^{T} \mathbf{A}\right) \mathbf{r}^{(0)}=\left(\mathbf{W}_{n-1}^{T} \mathbf{A}\right)\left(\sigma_{0} \mathbf{w}^{(0)}\right)
$$

$$
\begin{aligned}
& =\sigma_{0}\left(\mathbf{W}_{n-1}^{T} \mathbf{A} \mathbf{W}_{n-1}\right)\left(\mathbf{W}_{n-1}^{T} \mathbf{w}^{(0)}\right) \\
& =\sigma_{0} \mathbf{T}_{n} \mathbf{e}_{1},
\end{aligned}
$$

since $\mathbf{r}^{(0)}=\sigma_{0} \mathbf{w}^{(0)}, \mathbf{W}_{n-1} \mathbf{W}_{n-1}^{T}=\mathbf{I}$, and $\mathbf{W}_{n-1}^{T} \mathbf{A} \mathbf{W}_{n-1}=\mathbf{T}_{n}$. Here $\left\langle\mathbf{w}^{(i)}, \mathbf{w}^{(j)}\right\rangle=\delta_{i j}$. We obtain

$$
\begin{aligned}
\mathbf{T}_{n}\left(\mathbf{Q}_{n-1}\right) & =\left[\begin{array}{ccccccc}
\gamma_{0} & & & & & & \\
\delta_{1} & \gamma_{1} & & & & & \\
\varepsilon_{2} & \delta_{2} & \gamma_{2} & & & & \\
& \ddots & \ddots & \ddots & & & \\
& & \varepsilon_{n-3} & \delta_{n-3} & \gamma_{n-3} & & \\
& & & \varepsilon_{n-2} & \delta_{n-2} & \gamma_{n-2} & \\
& & & & \varepsilon_{n-1} & \delta_{n-1} & \bar{\gamma}_{n-1}
\end{array}\right]_{n \times n} \\
& \equiv \overline{\mathbf{L}}_{n},
\end{aligned}
$$

where

$$
\mathbf{Q}_{n-1} \equiv \mathbf{Q}_{1,2} \mathbf{Q}_{2,3} \mathbf{Q}_{3,4} \cdots \mathbf{Q}_{n-2, n-1} \mathbf{Q}_{n-1, n}
$$

Here a Givens rotation matrix is

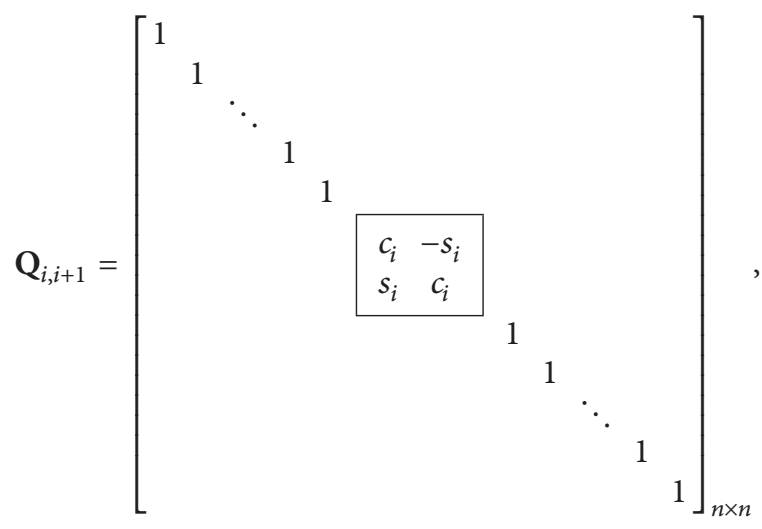

with $c_{i}^{2}+s_{i}^{2}=1$.

Here, we repeatedly apply Givens rotations to the righthand side of $\mathbf{T}_{n},(10)$, in order to zero out the $\beta_{i}$-diagonal above the $\alpha_{i}$ main diagonal and change the tridiagonal matrix $\mathbf{T}_{n}$ into a lower tridiagonal matrix $\overline{\mathbf{L}}_{n}$.

Then, from (22), we have

$$
\mathbf{T}_{n} \mathbf{Q}_{n-1}=\overline{\mathbf{L}}_{n} .
$$

Since $\mathbf{Q}_{n-1}^{-1}=\mathbf{Q}_{n-1}^{T}$, we obtain

$$
\mathbf{T}_{n}=\overline{\mathbf{L}}_{n} \mathbf{Q}_{n-1}^{T}, \quad \mathbf{T}_{n}^{T}=\mathbf{Q}_{n-1} \overline{\mathbf{L}}_{n}^{T} .
$$

Since $\mathbf{T}_{n}$ is symmetric, we have

$$
\begin{aligned}
\mathbf{T}_{n}^{2} & =\mathbf{T}_{n} \mathbf{T}_{n}=\mathbf{T}_{n} \mathbf{T}_{n}^{T} \\
& =\left(\overline{\mathbf{L}}_{n} \mathbf{Q}_{n-1}^{T}\right)\left(\mathbf{Q}_{n-1} \overline{\mathbf{L}}_{n}^{T}\right)=\overline{\mathbf{L}}_{n} \overline{\mathbf{L}}_{n}^{T} .
\end{aligned}
$$

Thus, we find that the coefficient matrix (26) can be written as

$$
\mathbf{T}_{n}^{2}+\sigma_{n}^{2} \mathbf{e}_{n} \mathbf{e}_{n}^{T}=\overline{\mathbf{L}}_{n} \overline{\mathbf{L}}_{n}^{T}+\sigma_{n}^{2} \mathbf{e}_{n} \mathbf{e}_{n}^{T} .
$$

Consequently, we are now interested in solving this linear system

$$
\left(\overline{\mathbf{L}}_{n} \overline{\mathbf{L}}_{n}^{T}+\sigma_{n}^{2} \mathbf{e}_{n} \mathbf{e}_{n}^{T}\right) \mathbf{y}^{(n)}=\sigma_{0} \mathbf{T}_{n} \mathbf{e}_{1}
$$

In the next step in the SYMMLQ method $[6,7]$, we solve

$$
\mathbf{T}_{n+1} \mathbf{y}^{(n+1)}=\sigma_{0} \mathbf{e}_{1} .
$$


Since

$$
\begin{aligned}
\left(\mathbf{T}_{n+1}\right) & \mathbf{Q}_{n-1} \\
& =\left[\begin{array}{cccccccc}
\gamma_{0} & & & & & & & \\
\delta_{1} & \gamma_{1} & & & & & \\
\varepsilon_{2} & \delta_{2} & \gamma_{2} & & & & & \\
& \ddots & \ddots & \ddots & & & & \\
& & \varepsilon_{n-3} & \delta_{n-3} & \gamma_{n-3} & & & \\
& & & \varepsilon_{n-2} & \delta_{n-2} & \gamma_{n-2} & & \\
& & & & \varepsilon_{n-1} & \delta_{n-1} & \bar{\gamma}_{n-1} & \sigma_{n} \\
& & & & & \varepsilon_{n} & \bar{\delta}_{n} & \alpha_{n}
\end{array}\right]_{(n+1) \times(n+1)},
\end{aligned}
$$

where

$$
Q_{n-1} \equiv \mathbf{Q}_{1,2} \mathbf{Q}_{2,3} \mathbf{Q}_{3,4} \cdots \mathbf{Q}_{n-3, n-2} \mathbf{Q}_{n-2, n-1} \mathbf{Q}_{n-1, n},
$$

then we choose

$$
c_{n}=\frac{\bar{\gamma}_{n-1}}{\gamma_{n-1}}, \quad \gamma_{n-1}=\sqrt{\bar{\gamma}_{n-1}^{2}+\sigma_{n}^{2}} .
$$

Thus, we have $\bar{\gamma}_{n-1}=c_{n} \gamma_{n-1}$. Recall that $c_{n}^{2}+s_{n}^{2}=1$ by (23). (For details on the SYMMLQ method, see $[6,7]$.)

Then, we have

$$
\begin{aligned}
& \mathbf{L}_{n}=\left[\begin{array}{ccccccc}
\gamma_{0} & & & & & & \\
\delta_{1} & \gamma_{1} & & & & & \\
\varepsilon_{2} & \delta_{2} & \gamma_{2} & & & & \\
& \ddots & \ddots & \ddots & & & \\
& & \varepsilon_{n-3} & \delta_{n-3} & \gamma_{n-3} & & \\
& & & \varepsilon_{n-2} & \delta_{n-2} & \gamma_{n-2} & \\
& & & & \varepsilon_{n-1} & \delta_{n-1} & \gamma_{n-1}
\end{array}\right]_{n \times n} \\
& =\left[\begin{array}{c} 
\\
\\
\end{array}\left[\begin{array}{c}
0 \\
0 \\
0 \\
\vdots \\
0 \\
\\
\gamma_{n-1}
\end{array}\right]_{n \times n}\right. \\
& =\mathbf{B}+\gamma_{n-1} \mathbf{e}_{n},
\end{aligned}
$$

where

$$
\mathbf{B}=\left[\begin{array}{cccccc}
\gamma_{0} & & & & & \\
\delta_{1} & \gamma_{1} & & & & \\
\varepsilon_{2} & \delta_{2} & \gamma_{2} & & & \\
& \ddots & \ddots & \ddots & & \\
& & \varepsilon_{n-3} & \delta_{n-3} & \gamma_{n-3} & \\
& & & \varepsilon_{n-2} & \delta_{n-2} & \gamma_{n-2} \\
& & & & \varepsilon_{n-1} & \delta_{n-1}
\end{array}\right]_{n \times(n-1)}
$$

(34)
Consequently, we obtain

$$
\begin{aligned}
\mathbf{L}_{n} \mathbf{L}_{n}^{T} & =\left(\mathbf{B}+\gamma_{n-1} \mathbf{e}_{n}\right)\left(\mathbf{B}^{T}+\gamma_{n-1} \mathbf{e}_{n}^{T}\right) \\
& =\mathbf{B} \mathbf{B}^{T}+\gamma_{n-1}^{2} \mathbf{e}_{n} \mathbf{e}_{n}^{T} .
\end{aligned}
$$

Then, we let

$$
\overline{\mathbf{L}}_{n} \equiv \mathbf{L}_{n} \widetilde{\mathbf{D}}_{n}
$$

where

$$
\widetilde{\mathbf{D}}_{n}=\left[\begin{array}{cccccc}
1 & & & & & \\
& 1 & & & & \\
& & \ddots & & \\
& & & 1 & & \\
& & & 1 & \\
& & & & c_{n}
\end{array}\right]_{n \times n} .
$$

So we have

$$
\overline{\mathbf{L}}=\mathbf{B}+\gamma_{n-1} c_{n} \mathbf{e}_{n}=\mathbf{B}+\bar{\gamma}_{n-1} \mathbf{e}_{n}
$$

since $\bar{\gamma}_{n-1}^{2} / \gamma_{n-1}^{2}=c_{n}$. Consequently, we have

$$
\begin{aligned}
\overline{\mathbf{L}}_{n} \overline{\mathbf{L}}_{n}^{T} & =\left(\mathbf{B}+\bar{\gamma}_{n-1} \mathbf{e}_{n}\right)\left(\mathbf{B}^{T}+\bar{\gamma}_{n-1} \mathbf{e}_{n}^{T}\right) \\
& =\mathbf{B B}^{T}+\bar{\gamma}_{n-1}^{2} \mathbf{e}_{n} \mathbf{e}_{n}^{T} .
\end{aligned}
$$

Using (35) and (39), we have

$$
\mathbf{L}_{n} \mathbf{L}_{n}^{T}-\gamma_{n-1}^{2} \mathbf{e}_{n} \mathbf{e}_{n}^{T}=\mathbf{B} \mathbf{B}^{T}=\overline{\mathbf{L}}_{n} \overline{\mathbf{L}}_{n}^{T}-\bar{\gamma}_{n-1}^{2} \mathbf{e}_{n} \mathbf{e}_{n}^{T}
$$

Since $\bar{\gamma}_{n-1}^{2}+\sigma_{n}^{2}=\gamma_{n-1}^{2}$, the coefficient matrix in linear equation (28) is

$$
\overline{\mathbf{L}}_{n} \overline{\mathbf{L}}_{n}^{T}+\sigma_{n}^{2} \mathbf{e}_{n} \mathbf{e}_{n}^{T}=\mathbf{L}_{n} \mathbf{L}_{n}^{T}
$$


From (24) and (36)

$$
\mathbf{T}_{n} \mathbf{Q}_{n-1}=\overline{\mathbf{L}}_{n}, \quad \overline{\mathbf{L}}_{n}=\mathbf{L}_{n} \widetilde{\mathbf{D}}_{n},
$$

we have the right-hand side vector in linear system (28)

$$
\begin{aligned}
\sigma_{0} \mathbf{T}_{n} \mathbf{e}_{1} & =\sigma_{0} \overline{\mathbf{L}}_{n} \mathbf{Q}_{n-1}^{T} \mathbf{e}_{1} \\
& =\sigma_{0} \mathbf{L}_{n} \widetilde{\mathbf{D}}_{n} \mathbf{Q}_{n-1}^{T} \mathbf{e}_{1} .
\end{aligned}
$$

Since $\mathbf{L}_{n}$ is nonsingular, and from (41) and (43), linear system (28) is

$$
\left(\mathbf{L}_{n} \mathbf{L}_{n}^{T}\right) \mathbf{y}^{(n)}=\sigma_{0} \mathbf{L}_{n} \widetilde{\mathbf{D}}_{n} \mathbf{Q}_{n-1}^{T} \mathbf{e}_{1}
$$

which reduces to

$$
\left(\mathbf{L}_{n}^{T}\right) \mathbf{y}^{(n)}=\sigma_{0} \widetilde{\mathbf{D}}_{n} \mathbf{Q}_{n-1}^{T} \mathbf{e}_{1}
$$

Thus, we obtain this equation for the $n$th iteration of the LQ MINRES method

$$
\begin{aligned}
\mathbf{u}_{\mathbf{L}}^{(n)} & =\mathbf{u}^{(0)}+\mathbf{W}_{n-1} \mathbf{y}^{(n)} \\
& =\mathbf{u}^{(0)}+\mathbf{W}_{n-1}\left(\mathbf{L}_{n}^{T}\right)^{-1}\left(\sigma_{0} \widetilde{\mathbf{D}}_{n} \mathbf{Q}_{n-1}^{T} \mathbf{e}_{1}\right) .
\end{aligned}
$$

\section{QR-MINRES Method}

Again, as in Section 3, we consider another method for solving linear system (18) for $\mathbf{y}^{(n)}$

$$
\left(\mathbf{W}_{n-1}^{T} \mathbf{A}^{2} \mathbf{W}_{n-1}\right) \mathbf{y}^{(n)}=\mathbf{W}_{n-1}^{T} \mathbf{A} \mathbf{r}^{(0)}
$$

Then, instead of solving linear system (47), we now solve

$$
\left(\mathbf{T}_{n}^{2}+\sigma_{n}^{2} \mathbf{e}_{n} \mathbf{e}_{n}^{T}\right) \mathbf{y}^{(n)}=\sigma_{0} \mathbf{T}_{n} \mathbf{e}_{1}
$$

First, we repeatedly apply Givens rotations to the lefthand side of $\mathbf{T}_{n}$

$$
\begin{aligned}
\mathbf{Q}_{n-1} \mathbf{T}_{n} & =\left[\begin{array}{ccccccc}
\gamma_{0} & \delta_{1} & \varepsilon_{2} & & & & \\
& \gamma_{1} & \delta_{2} & \varepsilon_{3} & & & \\
& & \gamma_{2} & \delta_{3} & \varepsilon_{4} & & \\
& & & \ddots & \ddots & \ddots & \\
& & & & \gamma_{n-3} & \delta_{n-2} & \varepsilon_{n-1} \\
& & & & & \gamma_{n-2} & \delta_{n-1} \\
& & & & & & \bar{\gamma}_{n-1}
\end{array}\right]_{n \times n} \\
& \equiv \overline{\mathbf{R}}_{n},
\end{aligned}
$$

where

$$
\mathbf{Q}_{n-1} \equiv \mathbf{Q}_{n-1, n} \mathbf{Q}_{n-2, n-1} \mathbf{Q}_{n-3, n-2} \cdots \mathbf{Q}_{3,4} \mathbf{Q}_{2,3} \mathbf{Q}_{1,2} .
$$

Here, we use Givens rotations applied on the left-hand side of $\mathbf{T}_{n}$, (10), to transform a tridiagonal matrix into an upper tridiagonal matrix $\overline{\mathbf{L}}_{n}$, (21).

Then, we obtain

$$
\mathbf{Q}_{n-1} \mathbf{T}_{n}=\overline{\mathbf{R}}_{n}, \quad \mathbf{T}_{n}=\mathbf{Q}_{n-1}^{T} \overline{\mathbf{R}}_{n} .
$$

Since $\mathbf{T}_{n}$ is symmetric, we obtain

$$
\mathbf{T}_{n}=\mathbf{T}_{n}^{T}=\overline{\mathbf{R}}_{n}^{T} \mathbf{Q}_{n-1} .
$$

Thus, the coefficient matrix in linear system (48) can be written as

$$
\mathbf{T}_{n}^{2}+\sigma_{n}^{2} \mathbf{e}_{n} \mathbf{e}_{n}^{T}=\overline{\mathbf{R}}_{n}^{T} \overline{\mathbf{R}}_{n}+\sigma_{n}^{2} \mathbf{e}_{n} \mathbf{e}_{n}^{T} .
$$

Consequently, we are interested in solving this linear system

$$
\left(\overline{\mathbf{R}}_{n} \overline{\mathbf{R}}_{n}^{T}+\sigma_{n}^{2} \mathbf{e}_{n} \mathbf{e}_{n}^{T}\right) \mathbf{y}^{(n)}=\sigma_{0} \mathbf{T}_{n} \mathbf{e}_{1} .
$$

In the next step in the SYMMQR method $[6,7]$, we solve this linear system

$$
\mathbf{T}_{n+1} \mathbf{y}^{(n+1)}=\sigma_{0} \mathbf{e}_{1}
$$

Since

$$
\left(\mathbf{Q}_{n-1}\right) \mathbf{T}_{n+1}
$$

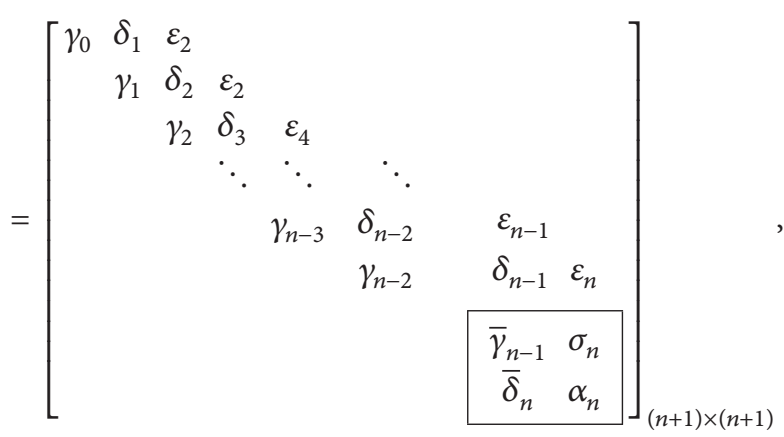


where

$$
\mathbf{Q}_{n-1} \equiv \mathbf{Q}_{n-1, n} \mathbf{Q}_{n-2, n-1} \mathbf{Q}_{n-3, n-2} \cdots \mathbf{Q}_{2,3} \mathbf{Q}_{1,2},
$$

then we choose

$$
c_{n}=\frac{\bar{\gamma}_{n-1}}{\gamma_{n-1}}, \quad \gamma_{n-1}=\sqrt{\bar{\gamma}_{n-1}^{2}+\sigma_{n}^{2}} .
$$

Thus, we obtain $\bar{\gamma}_{n-1}=c_{n} \gamma_{n-1}$. (For details on the SYMMQR method, see $[6,7]$.)

Let

$$
\begin{aligned}
& \mathbf{R}_{n}=\left[\begin{array}{ccccccc}
\gamma_{0} & \delta_{1} & \varepsilon_{2} & & & & \\
& \gamma_{1} & \delta_{2} & \varepsilon_{3} & & & \\
& & \gamma_{2} & \delta_{3} & \varepsilon_{4} & & \\
& & & \ddots & \ddots & \ddots & \\
& & & & \gamma_{n-3} & \delta_{n-2} & \varepsilon_{n-1} \\
& & & & & \gamma_{n-2} & \delta_{n-1} \\
& & & & & & \gamma_{n-1}
\end{array}\right]_{n \times n} \\
& =\left[\begin{array}{ccccccc}
\multicolumn{6}{c}{\mathbf{B}} & \\
\hline 0 & 0 & 0 & \cdots & 0 & 0 & \gamma_{n-1}
\end{array}\right]_{n \times n} \\
& =\mathbf{B}+\gamma_{n-1} \mathbf{e}_{n}^{T},
\end{aligned}
$$

where

$$
\mathbf{B}=\left[\begin{array}{ccccccc}
\gamma_{0} & \delta_{1} & \varepsilon_{2} & & & & \\
& \gamma_{1} & \delta_{2} & \varepsilon_{3} & & & \\
& & \gamma_{2} & \delta_{3} & \varepsilon_{4} & & \\
& & & \ddots & \ddots & \ddots & \\
& & & & \gamma_{n-3} & \delta_{n-2} & \varepsilon_{n-1} \\
& & & & & \gamma_{n-2} & \delta_{n-1}
\end{array}\right]_{(n-1) \times n}
$$

We have

$$
\begin{aligned}
\mathbf{R}_{n}^{T} \mathbf{R}_{n} & =\left(\mathbf{B}^{T}+\gamma_{n-1} \mathbf{e}_{n}\right)\left(\mathbf{B}+\gamma_{n-1} \mathbf{e}_{n}^{T}\right) \\
& =\mathbf{B}^{T} \mathbf{B}+\gamma_{n-1}^{2} \mathbf{e}_{n} \mathbf{e}_{n}^{T} .
\end{aligned}
$$

Then, we have

$$
\overline{\mathbf{R}}_{n}=\widetilde{\mathbf{D}}_{n} \mathbf{R}_{n}
$$

where

$$
\widetilde{\mathbf{D}}_{n}=\left[\begin{array}{llllll}
1 & & & & & \\
& 1 & & & & \\
& & \ddots & & & \\
& & & 1 & & \\
& & & & 1 & \\
& & & & & c_{n}
\end{array}\right]_{n \times n} .
$$

So we have

$$
\begin{aligned}
& \overline{\mathbf{R}}_{n}=\mathbf{B}+c_{n} \gamma_{n-1} \mathbf{e}_{n}^{T}=\mathbf{B}+\bar{\gamma}_{n-1} \mathbf{e}_{n}^{T}, \\
& \overline{\mathbf{R}}_{n}^{T}=\mathbf{B}^{T}+\bar{\gamma}_{n-1} \mathbf{e}_{n},
\end{aligned}
$$

since $\bar{\gamma}_{n-1} / \gamma_{n-1}=c_{n}$. Consequently, we obtain

$$
\begin{aligned}
\overline{\mathbf{R}}_{n}^{T} \overline{\mathbf{R}}_{n} & =\left(\mathbf{B}^{T}+\bar{\gamma}_{n-1} \mathbf{e}_{n}\right)\left(\mathbf{B}+\bar{\gamma}_{n-1} \mathbf{e}_{n}\right)^{T} \\
& =\mathbf{B}^{T} \mathbf{B}+\bar{\gamma}_{n-1}^{2} \mathbf{e}_{n} \mathbf{e}_{n}^{T} .
\end{aligned}
$$

Using (64), we have

$$
\mathbf{R}_{n} \mathbf{R}_{n}^{T}-\gamma_{n}^{2} \mathbf{e}_{n} \mathbf{e}_{n}^{T}=\overline{\mathbf{R}}_{n} \overline{\mathbf{R}}_{n}^{T}-\bar{\gamma}_{n}^{2} \mathbf{e}_{n} \mathbf{e}_{n}^{T} .
$$

Since $\bar{\gamma}_{n-1}^{2}+\sigma_{n}^{2}=\gamma_{n-1}^{2}$, the coefficient matrix in the linear equation (54) is

$$
\overline{\mathbf{R}}_{n} \overline{\mathbf{R}}_{n}^{T}+\sigma_{n}^{2} \mathbf{e}_{n} \mathbf{e}_{n}^{T}=\mathbf{R}_{n} \mathbf{R}_{n}^{T} .
$$

Since

$$
\mathbf{T}_{n}=\overline{\mathbf{R}}_{n}^{T} \mathbf{Q}_{n-1}, \quad \overline{\mathbf{R}}_{n}=\widetilde{\mathbf{D}}_{n} \mathbf{R}_{n},
$$

we have right-hand side vector in the linear system (54)

$$
\begin{aligned}
\sigma_{0} \mathbf{T}_{n} \mathbf{e}_{1} & =\sigma_{0} \overline{\mathbf{R}}_{n}^{T} \mathbf{Q}_{n-1} \mathbf{e}_{1} \\
& =\sigma_{0} \mathbf{R}_{n}^{T} \widetilde{\mathbf{D}}_{n} \mathbf{Q}_{n-1} \mathbf{e}_{1} .
\end{aligned}
$$

Since $\mathbf{R}_{n}$ is nonsingular, we obtain the linear system (54)

$$
\left(\mathbf{R}_{n}^{T} \mathbf{R}_{n}\right) \mathbf{y}^{(n)}=\sigma_{0} \mathbf{R}_{n}^{T} \widetilde{\mathbf{D}}_{n} \mathbf{Q}_{n-1} \mathbf{e}_{1},
$$

which reduces to

$$
\mathbf{R}_{n} \mathbf{y}^{(n)}=\sigma_{0} \widetilde{\mathbf{D}}_{n} \mathbf{Q}_{n-1} \mathbf{e}_{1} .
$$

Thus, we obtain the equation for the $n$th iteration of the QRMINRES method

$$
\begin{aligned}
\mathbf{u}_{\mathbf{R}}^{(n)} & =\mathbf{u}^{(0)}+\mathbf{W}_{n-1} \mathbf{y}^{(n)} \\
& =\mathbf{u}^{(0)}+\mathbf{W}_{n-1} \mathbf{R}_{n}^{-1}\left(\sigma_{0} \widetilde{\mathbf{D}}_{n} \mathbf{Q}_{n-1} \mathbf{e}_{1}\right) .
\end{aligned}
$$

\section{Relation between LQ-MINRES and QR-MINRES}

Now, we show that the LQ-MINRES method and the QRMINRES method are essentially the same. In the LQMINRES method (46), we have

$$
\mathbf{u}_{\mathbf{L}}^{(n)}=\mathbf{u}^{(0)}+\mathbf{W}_{n-1}\left(\mathbf{L}_{n}^{T}\right)^{-1}\left(\sigma_{0} \widetilde{\mathbf{D}}_{n}^{\mathbf{L}} \mathbf{Q}_{\mathbf{L}}^{T} \mathbf{e}_{1}\right) .
$$

In the QR-MINRES method (72), we have

$$
\mathbf{u}_{\mathbf{R}}^{(n)}=\mathbf{u}^{(0)}+\mathbf{W}_{n-1} \mathbf{R}_{n}^{-1}\left(\sigma_{0} \widetilde{\mathbf{D}}_{n}^{\mathbf{R}} \mathbf{Q}_{\mathbf{R}} \mathbf{e}_{1}\right) .
$$


For the LQ-MINRES method (73), we have

$$
\begin{aligned}
& c_{1}=\frac{\alpha_{0}}{\gamma_{0}}, \quad c_{k}^{\mathrm{L}}=\frac{\bar{\gamma}_{k-1}^{\mathrm{L}}}{\gamma_{k-1}^{\mathrm{L}},} \\
& s_{1}=\frac{\beta_{1}}{\gamma_{0}}, \quad s_{k}^{\mathbf{L}}=\frac{\beta_{k}}{\gamma_{k-1}^{\mathbf{L}}}, \\
& \bar{\delta}_{k}^{\mathbf{L}}=\left\{\begin{array}{l}
\beta_{1}, \quad k=1, \\
-c_{k-1}^{\mathbf{L}} \beta_{k},
\end{array}\right. \\
& \delta_{k}^{\mathbf{L}}=c_{k}^{\mathbf{L}} \bar{\delta}_{k}^{\mathbf{L}}+s_{k}^{\mathbf{L}} \alpha_{k} \text {, } \\
& \varepsilon_{k}^{\mathrm{L}}=s_{k-1}^{\mathbf{L}} \beta_{k} \text {, } \\
& \bar{\gamma}_{k}^{\mathbf{L}}= \begin{cases}\alpha_{0}, & k=0, \\
-s_{k}^{\mathbf{L}} \bar{\delta}_{k}^{\mathbf{L}}-c_{k}^{\mathbf{L}} \alpha_{k}, & \end{cases} \\
& \gamma_{k}^{\mathbf{L}}=\sqrt{\left(\bar{\gamma}_{k}^{\mathbf{L}}\right)^{2}+\sigma_{k+1}^{2}} .
\end{aligned}
$$

In the QR-MINRES method (46), we have

$$
\begin{aligned}
& c_{1}=\frac{\alpha_{0}}{\gamma_{0}}, \quad c_{k}^{\mathbf{R}}=\frac{\bar{\gamma}_{k-1}^{\mathbf{R}}}{\gamma_{k-1}^{\mathbf{R}}}, \\
& s_{1}=-\frac{\beta_{1}}{\gamma_{0}}, \quad s_{k}^{\mathbf{R}}=-\frac{\beta_{k}}{\gamma_{k-1}^{\mathbf{R}}}, \\
& \bar{\delta}_{k}^{\mathbf{R}}=\left\{\begin{array}{l}
\beta_{1}, \\
c_{k-1}^{\mathbf{R}} \beta_{k},
\end{array}\right. \\
& \delta_{k}^{\mathbf{R}}=c_{k}^{\mathbf{R}} \bar{\delta}_{k}^{\mathbf{R}}-s_{k}^{\mathbf{R}} \alpha_{k}, \\
& \varepsilon_{k}^{\mathbf{R}}=-s_{k-1}^{\mathbf{R}} \beta_{k}, \\
& \bar{\gamma}_{k}^{\mathbf{R}}= \begin{cases}\alpha_{0}, & k=0, \\
s_{k}^{\mathbf{R}} \bar{\delta}_{k}^{\mathbf{R}}+c_{k}^{\mathbf{R}} \alpha_{k}, & \end{cases} \\
& \gamma_{k}^{\mathbf{R}}=\sqrt{\left(\bar{\gamma}_{k}^{\mathbf{R}}\right)^{2}+\sigma_{k+1}^{2}} .
\end{aligned}
$$

From the computation and by induction, we have the following relations between the LQ-MINRES method and the QRMINRES method:

$$
\begin{aligned}
c_{k}^{\mathbf{L}} & = \begin{cases}c_{k}^{\mathbf{R}}, & k: \text { odd, } \\
-c_{k}^{\mathbf{R}}, & k: \text { even, }\end{cases} \\
\bar{\gamma}_{k}^{\mathbf{L}} & = \begin{cases}-\gamma_{k}^{\mathbf{R}}, & k: \text { odd, } \\
\gamma_{k}^{\mathbf{R}}, & k: \text { even, }\end{cases} \\
\bar{\delta}_{k}^{\mathbf{L}} & = \begin{cases}\delta_{k}^{\mathbf{R}}, & k: \text { odd, } \\
-\delta_{k}^{\mathbf{R}}, & k: \text { even, }\end{cases} \\
s_{k}^{\mathbf{L}} & =-s_{k}^{\mathbf{R}} .
\end{aligned}
$$

By induction, we have

$$
\delta_{k}^{\mathbf{L}}=\delta_{k}^{\mathbf{R}}, \quad \varepsilon_{k}^{\mathbf{L}}=\varepsilon_{k}^{\mathbf{R}}, \quad \gamma_{k}^{\mathrm{L}}=\gamma_{k}^{\mathbf{R}} .
$$

Hence, we obtain

$$
\mathbf{L}_{n}^{T}=\mathbf{R}_{n}
$$

Moreover, if we let

$$
\begin{aligned}
& \sigma_{0} \widetilde{\mathbf{D}}_{n}^{\mathbf{L}} \mathbf{Q}_{\mathbf{L}}^{T} \mathbf{e}_{1}=\left[\xi_{0}^{\mathbf{L}}, \xi_{1}^{\mathbf{L}}, \xi_{2}^{\mathbf{L}}, \ldots, \xi_{n-3}^{\mathbf{L}}, \xi_{n-2}^{\mathbf{L}}, \xi_{n-1}^{\mathbf{L}}\right]_{1 \times n}^{T}, \\
& \sigma_{0} \widetilde{\mathbf{D}}_{n}^{\mathbf{R}} \mathbf{Q}_{\mathbf{R}} \mathbf{e}_{1}=\left[\xi_{0}^{\mathbf{R}}, \xi_{1}^{\mathbf{R}}, \xi_{2}^{\mathbf{R}}, \ldots, \xi_{n-3}^{\mathbf{R}}, \xi_{n-2}^{\mathbf{R}}, \xi_{n-1}^{\mathbf{R}}\right]_{1 \times n}^{T},
\end{aligned}
$$

then

$$
\begin{aligned}
\xi_{i}^{\mathbf{L}} & =c_{i+1}^{\mathbf{L}} s_{1}^{\mathbf{L}} s_{2}^{\mathbf{L}} s_{3}^{\mathbf{L}} \cdots s_{i-2}^{\mathbf{L}} s_{i-1}^{\mathbf{L}} s_{i}^{\mathbf{L}} \sigma_{0} \\
& =c_{i+1}^{\mathbf{L}}(-1)^{i} s_{1}^{\mathbf{R}} s_{2}^{\mathbf{R}} s_{3}^{\mathbf{R}} \cdots s_{s-2}^{\mathbf{R}} s_{i-1}^{\mathbf{R}} s_{i}^{\mathbf{R}} \sigma_{0} \\
& =c_{i+1}^{\mathbf{R}} s_{1}^{\mathbf{R}} s_{2}^{\mathbf{R}} s_{3}^{\mathbf{R}} \cdots s_{i-2}^{\mathbf{R}} s_{i-1}^{\mathbf{R}} s_{i}^{\mathbf{R}} \sigma_{0} \\
& =\xi_{i}^{\mathbf{R}} .
\end{aligned}
$$

Thus, we obtain

$$
\mathbf{u}_{\mathbf{L}}^{(n)}=\mathbf{u}_{\mathbf{R}}^{(n)}
$$

\section{A Closer Look at QR-MINRES and SYMMQR}

In the SYMMQR method [6, 7], we have two estimated solutions: $\mathbf{u}_{\mathrm{ls}}^{(n)}$ and $\mathbf{u}_{\mathrm{Gal}}^{(n)}$.

The first estimated solution is

$$
\mathbf{u}_{1 \mathrm{~s}}^{(n)}=\mathbf{u}^{(0)}+\mathbf{W}_{n-1} \mathbf{y}_{\mathrm{ls}}^{(n)},
$$

where $\mathbf{y}_{\mathrm{ls}}^{(n)}$ is the solution of this linear system

$$
\mathbf{R}_{n} \mathbf{y}^{(n)}=\mathbf{z}^{(n)},
$$

with the right-hand side vector being

$$
\mathbf{z}^{(n)}=\left[\xi_{0}, \xi_{1}, \xi_{2}, \ldots, \xi_{n-3}, \xi_{n-2}, \xi_{n-1}\right]_{1 \times n}^{T} .
$$

From these equations, we have

$$
\mathbf{z}^{(n)}=\sigma_{0} \widetilde{\mathbf{D}}_{n}^{\mathbf{R}} \mathbf{Q}_{\mathbf{R}} \mathbf{e}_{1} .
$$

Thus, we have

$$
\mathbf{u}_{\mathbf{R}}^{(n)}=\mathbf{u}_{\mathrm{ls}}^{(n)},
$$

where $\mathbf{u}_{\mathrm{ls}}^{(n)}$ is the solution of this least square problem

$$
\min _{\mathbf{y}^{(n)} \in \mathbb{R}^{n}}\left\|\widetilde{\mathbf{T}}_{n+1} \mathbf{y}^{(n)}-\sigma_{0} \mathbf{e}_{1}\right\|
$$

The second estimated solution is

$$
\mathbf{u}_{\mathrm{Gal}}^{(n)}=\mathbf{u}^{(0)}+\mathbf{W}_{n-1} \mathbf{y}_{\mathrm{Gal}}^{(n)},
$$

where $\mathbf{y}_{\mathrm{Gal}}$ is the solution of this linear system

$$
\begin{gathered}
\overline{\mathbf{R}}_{n} \mathbf{y}^{(n)}=\overline{\mathbf{z}}^{(n)}, \\
\mathbf{u}_{\mathrm{Gal}}^{(n)}-\mathbf{u}^{(0)}=\widetilde{\mathbf{D}}_{n}^{-2}\left(\mathbf{u}_{\mathbf{R}}^{(n)}-\mathbf{u}^{(0)}\right) .
\end{gathered}
$$




\section{Modified MINRES (MMINRES) Method}

Next, we assume that matrix $\mathbf{A}$ is nonsymmetric. In $[6,7]$, we use a long term recurrence to generate orthonormal vectors $\left\{\mathbf{w}^{(0)}, \mathbf{w}^{(1)}, \mathbf{w}^{(2)}, \ldots, \mathbf{w}^{(n-3)}, \mathbf{w}^{(n-2)}, \mathbf{w}^{(n-1)}\right\}$ as follows:

$$
\begin{aligned}
& \mathbf{u}^{(0)}=\text { arbitrary, } \\
& \mathbf{r}^{(0)}=\mathbf{b}-\mathbf{A} \mathbf{u}^{(0)}, \\
& \widetilde{\mathbf{w}}^{(0)}=\mathbf{r}^{(0)}, \\
& \sigma_{0}=\sqrt{\left\langle\widetilde{\mathbf{w}}^{(0)}, \widetilde{\mathbf{w}}^{(0)}\right\rangle}, \\
& \mathbf{w}^{(0)}=\left(\frac{1}{\sigma_{0}}\right) \widetilde{\mathbf{w}}^{(0)}, \\
& \text { for } i=1,2, \ldots, n-1 \text {, } \\
& \widetilde{\mathbf{w}}^{(i)}=\mathbf{A} \mathbf{w}^{(i-1)} \sum_{j=0}^{i-1} \mathbf{b}_{i, j} \mathbf{w}^{(j)}, \\
& \text { where } \mathbf{b}_{i, j}=\left\langle\mathbf{A} \mathbf{w}^{(i-1)}, \mathbf{w}^{(j)}\right\rangle \text {, } \\
& \sigma_{i}=\sqrt{\left\langle\widetilde{\mathbf{w}}^{(i)}, \widetilde{\mathbf{w}}^{(i)}\right\rangle}, \\
& \mathbf{w}^{(i)}=\left(\frac{1}{\sigma_{i}}\right) \widetilde{\mathbf{w}}^{(i)},
\end{aligned}
$$

end for.

Consequently, we obtain the following matrix equations:

$$
\begin{aligned}
\mathbf{A W}_{n-1} & =\mathbf{W}_{n-1} \mathbf{H}_{n}+\sigma_{n} \mathbf{w}^{(n)} \mathbf{e}_{n}^{T} \\
& =\mathbf{W}_{n} \widetilde{\mathbf{H}}_{n+1}, \\
& \mathbf{W}_{n-1}^{T} \mathbf{W}_{n-1}=\mathbf{I},
\end{aligned}
$$

where

$$
\begin{aligned}
& \mathbf{W}_{n-1}=\left[\mathbf{w}^{(0)}, \mathbf{w}^{(1)}, \mathbf{w}^{(2)}, \ldots, \mathbf{w}^{(n-3)}, \mathbf{w}^{(n-2)}, \mathbf{w}^{(n-1)}\right]_{n \times n}, \\
& \mathbf{e}_{n}^{T}=[0,0,0, \ldots, 0,0,0,1]_{1 \times n}, \\
& \mathbf{H}_{n}=\left[\begin{array}{ccccccc}
b_{1,0} & b_{2,0} & b_{3,0} & \cdots & b_{n-2,0} & b_{n-1,0} & b_{n, 0} \\
\sigma_{1} & b_{2,1} & b_{3,1} & \cdots & b_{n-2,1} & b_{n-1,1} & b_{n, 1} \\
& \sigma_{2} & b_{3,2} & \cdots & b_{n-2,2} & b_{n-1,2} & b_{n, 2} \\
& & \ddots & \ddots & \vdots & \vdots & \vdots \\
& & & \sigma_{n-3} & b_{n-2, n-3} & b_{n-1, n-3} & b_{n, n-3} \\
& & & & \sigma_{n-2} & b_{n-1, n-2} & b_{n, n-2} \\
& & & & & \sigma_{n-1} & b_{n, n-1}
\end{array}\right]_{n \times n}
\end{aligned}
$$

$\widetilde{\mathbf{H}}_{n+1}$

$$
=\left[\begin{array}{ccccccc}
b_{1,0} & b_{2,0} & b_{3,0} & \cdots & b_{n-2,0} & b_{n-1,0} & b_{n, 0} \\
\sigma_{1} & b_{2,1} & b_{3,1} & \cdots & b_{n-2,1} & b_{n-1,1} & b_{n, 1} \\
& \sigma_{2} & b_{3,2} & \cdots & b_{n-2,2} & b_{n-1,2} & b_{n, 2} \\
& & \ddots & \ddots & \vdots & \vdots & \vdots \\
& & & \sigma_{n-3} & b_{n-2, n-3} & b_{n-1, n-3} & b_{n, n-3} \\
& & & & \sigma_{n-2} & b_{n-1, n-2} & b_{n, n-2} \\
& & & & & \sigma_{n-1} & b_{n, n-1} \\
& & & & & & \sigma_{n}
\end{array}\right]_{(n+1) \times n}
$$

Since the matrix $\mathbf{H}_{n}$ is a full upper Hessenberg matrix, the LQ-MINRES method is not a practical procedure. Hence, we discuss only a generalization of the QR-MINRES method.

\section{Generalized QR-MINRES Method}

Since the matrix $\mathbf{A}$ is nonsymmetric, to minimize an expression such as this

$$
\min _{\mathbf{y}^{(n)} \in \mathbb{R}^{n}} f\left(\mathbf{y}^{(n)}\right)=\left(\mathbf{r}^{(n)}\right)^{T}\left(\mathbf{r}^{(n)}\right)
$$

we choose $\mathbf{y}^{(n)}$ to satisfy

$$
\left(\mathbf{W}_{n-1}^{T} \mathbf{A}^{T} \mathbf{A} \mathbf{W}_{n-1}\right) \mathbf{y}^{(n)}=\mathbf{W}_{n-1}^{T} \mathbf{A}^{T} \mathbf{r}^{(0)} .
$$

First, from the Arnoldi process and from the left-hand side of linear system (96), we can write the coefficient matrix of this linear system as

$$
\begin{aligned}
& \mathbf{W}_{n-1}^{T} \mathbf{A}^{T} \mathbf{A} \mathbf{W}_{n-1} \\
& =\left(\mathbf{A W}_{n-1}\right)^{T} \mathbf{A} \mathbf{W}_{n-1} \\
& =\left(\mathbf{W}_{n-1} \mathbf{H}_{n}+\sigma_{n} \mathbf{w}^{(n)} \mathbf{e}_{n}^{T}\right)^{T}\left(\mathbf{W}_{n-1} \mathbf{H}_{n}+\sigma_{n} \mathbf{w}^{(n)} \mathbf{e}_{n}^{T}\right) \\
& =\mathbf{H}_{n}^{T} \mathbf{H}_{n}+\sigma_{n}^{2} \mathbf{e}_{n} \mathbf{e}_{n}^{T} .
\end{aligned}
$$

Second, from the right-hand side vector of the linear system (96), we have

$$
\begin{aligned}
\left(\mathbf{W}_{n-1}^{T} \mathbf{A}^{T}\right) \mathbf{r}^{(0)} & \\
= & \left(\mathbf{A W}_{n-1}\right)^{T} \sigma_{0} \mathbf{w}^{(0)} \\
& =\left(\mathbf{W}_{n-1} \mathbf{H}_{n}+\sigma_{n} \mathbf{w}^{(n)} \mathbf{e}_{n}^{T}\right)^{T} \sigma_{0} \mathbf{w}^{(0)} \\
& =\left(\mathbf{H}_{n}^{T} \mathbf{W}_{n-1}^{T}\right) \sigma_{0} \mathbf{w}^{(0)} \\
& =\sigma_{0} \mathbf{H}_{n}^{T} \mathbf{e}_{1} .
\end{aligned}
$$


Consequently, instead of solving (96), we solve this linear system

$$
\left(\mathbf{H}_{n}^{T} \mathbf{H}_{n}+\sigma_{n}^{2} \mathbf{e}_{n} \mathbf{e}_{n}^{T}\right) \mathbf{y}^{(n)}=\sigma_{0} \mathbf{H}_{n}^{T} \mathbf{e}_{1}
$$

First, we repeatedly apply Givens rotations to the lefthand side of $\mathbf{H}_{n}$

$$
\mathbf{Q}_{n-1, n} \mathbf{Q}_{n-2, n-1} \mathbf{Q}_{n-3, n-2} \cdots \mathbf{Q}_{3,4} \mathbf{Q}_{2,3} \mathbf{Q}_{1,2} \mathbf{H}_{n} \equiv \overline{\mathbf{R}}_{n}
$$

By defining

$$
\mathbf{Q}_{n-1} \equiv \mathbf{Q}_{n-1, n} \mathbf{Q}_{n-2, n-1} \mathbf{Q}_{n-3, n-2} \cdots \mathbf{Q}_{3,4} \mathbf{Q}_{2,3} \mathbf{Q}_{1,2} \text {, }
$$

we obtain

$$
\begin{aligned}
& \mathbf{Q}_{n-1} \mathbf{H}_{n}=\overline{\mathbf{R}}_{n}, \\
& \mathbf{H}_{n}=\mathbf{Q}_{n-1}^{T} \overline{\mathbf{R}}_{n},
\end{aligned}
$$

so that

$$
\mathbf{H}_{n}^{T}=\overline{\mathbf{R}}_{n}^{T} \mathbf{Q}_{n-1} \text {. }
$$

Thus, the coefficient matrix in linear system (99) has the following form by using (103) and (104):

$$
\begin{aligned}
\mathbf{H}_{n}^{T} \mathbf{H}_{n}+\sigma_{n}^{2} \mathbf{e}_{n} \mathbf{e}_{n}^{T} & =\overline{\mathbf{R}}_{n}^{T} \overline{\mathbf{R}}_{n}+\sigma_{n}^{2} \mathbf{e}_{n} \mathbf{e}_{n}^{T} \\
& =\mathbf{R}_{n}^{T} \mathbf{R}_{n}+\sigma_{n}^{2} \mathbf{e}_{n} \mathbf{e}_{n}^{T} .
\end{aligned}
$$

Moreover, by using (102) and (104), the right-hand side vector in linear system (99) is

$$
\begin{aligned}
\sigma_{0} \mathbf{H}_{n}^{T} \mathbf{e}_{1} & =\sigma_{0} \overline{\mathbf{R}}_{n}^{T} \mathbf{Q}_{n-1} \mathbf{e}_{1} \\
& =\sigma_{0} \mathbf{R}_{n}^{T} \widetilde{\mathbf{D}}_{n} \mathbf{Q}_{n-1} \mathbf{e}_{1},
\end{aligned}
$$

where

$$
\begin{aligned}
& \mathbf{R}_{n}=\left[\begin{array}{ccccccc}
\gamma_{0} & \times & \times & \cdots & \cdots & \cdots & \times \\
& \gamma_{1} & \times & \times & \cdots & \cdots & \times \\
& & \gamma_{2} & \times & \times & \cdots & \times \\
& & & \ddots & \ddots & \ddots & \vdots \\
& & & & \gamma_{n-3} & \times & \times \\
& & & & \gamma_{n-2} & \times \\
& & & & & \gamma_{n-1}
\end{array}\right]_{n \times n} \\
& \widetilde{\mathbf{D}}_{n}=\left[\begin{array}{cccccc}
1 & & & & \\
& 1 & & & \\
& & \ddots & & \\
& & & 1 & & \\
& & & & 1 & \\
& & & & & c_{n}
\end{array}\right]_{n \times n},
\end{aligned}
$$

where $c_{n}=\bar{\gamma}_{n-1} / \gamma_{n-1}$ and $\gamma_{n-1}=\sqrt{\bar{\gamma}_{n-1}^{2}+\sigma_{n}^{2}}$. Thus, we obtain $\bar{\gamma}_{n-1}=c_{n} \gamma_{n-1}$. Then, we have

$$
\overline{\mathbf{R}}_{n}=\widetilde{\mathbf{D}}_{n} \mathbf{R}_{n}
$$

Let

$$
\mathbf{B} \equiv\left[\begin{array}{ccccccc}
\gamma_{0} & \times & \times & \times & \times & \times & \times \\
& \gamma_{1} & \ddots & \ddots & & & \vdots \\
& & \gamma_{2} & \ddots & \ddots & & \vdots \\
& & & \ddots & \ddots & \ddots & \vdots \\
& & & & \gamma_{n-3} & \ddots & \times \\
& & & & \gamma_{n-2} & \times
\end{array}\right]_{(n-1) \times n}
$$

Then, we have

$$
\mathbf{R}_{n}=\mathbf{B}+\gamma_{n-1} \mathbf{e}_{n}
$$

and we obtain

$$
\begin{aligned}
\mathbf{R}_{n}^{T} \mathbf{R}_{n} & =\left(\mathbf{B}^{T}+\gamma_{n-1} \mathbf{e}_{n}^{T}\right)\left(\mathbf{B}+\gamma_{n-1} \mathbf{e}_{n}\right) \\
& =\mathbf{B}^{T} \mathbf{B}+\gamma_{n-1}^{2} \mathbf{e}_{n}^{T} \mathbf{e}_{n} .
\end{aligned}
$$

We have

$$
\begin{aligned}
\overline{\mathbf{R}}_{n} & =\widetilde{\mathbf{D}}_{n} \mathbf{R}_{n} \\
& =\mathbf{B}+c_{n} \gamma_{n-1} \mathbf{e}_{n} \\
& =\mathbf{B}+\bar{\gamma}_{n-1} \mathbf{e}_{n}, \\
\overline{\mathbf{R}}_{n}^{T} \overline{\mathbf{R}}_{n} & =\left(\mathbf{B}^{T}+\bar{\gamma}_{n-1} \mathbf{e}_{n}^{T}\right)\left(\mathbf{B}+\bar{\gamma}_{n-1} \mathbf{e}_{n}\right) \\
& =\mathbf{B}^{T} \mathbf{B}+\bar{\gamma}^{2} \mathbf{e}_{n}^{T} \mathbf{e}_{n} .
\end{aligned}
$$

Using $\bar{\gamma}_{n-1}^{2}+\sigma_{n}^{2}=\gamma_{n}^{2}$, (112), and (114) we find that the coefficient matrix in linear system (104) is

$$
\overline{\mathbf{R}}_{n}^{T} \overline{\mathbf{R}}_{n}+\sigma_{n}^{2} \mathbf{e}_{n} \mathbf{e}_{n}^{T}=\mathbf{R}_{n}^{T} \mathbf{R}_{n}
$$

and the right-hand side vector is

$$
\begin{aligned}
\sigma_{0} \mathbf{H}_{n}^{T} \mathbf{e}_{1} & =\sigma_{0} \overline{\mathbf{R}}_{n}^{T} \mathbf{Q}_{n-1} \mathbf{e}_{1} \\
& =\sigma_{0} \mathbf{R}_{n}^{T} \widetilde{\mathbf{D}}_{n} \mathbf{Q}_{n-1} \mathbf{e}_{1} .
\end{aligned}
$$

Since $\mathbf{R}_{n}$ is nonsingular, we obtain this linear system

$$
\left(\mathbf{R}_{n}^{T} \mathbf{R}_{n}\right) \mathbf{y}^{(n)}=\sigma_{0} \mathbf{R}_{n}^{T} \widetilde{\mathbf{D}}_{n} \mathbf{Q}_{n-1} \mathbf{e}_{1},
$$

which reduces to this linear system

$$
\mathbf{R}_{n} \mathbf{y}^{(n)}=\sigma_{0} \widetilde{\mathbf{D}}_{n} \mathbf{Q}_{n-1} \mathbf{e}_{1}
$$


Thus, we obtain the $n$th iteration of the generalized QRMINRES method

$$
\begin{aligned}
\mathbf{u}_{\mathbf{R}}^{(n)} & =\mathbf{u}^{(0)}+\mathbf{W}_{n-1} \mathbf{y}^{(n)} \\
& =\mathbf{u}^{(0)}+\mathbf{W}_{n-1} \mathbf{R}_{n}^{-1}\left(\sigma_{0} \widetilde{\mathbf{D}}_{n} \mathbf{Q}_{n-1} \mathbf{e}_{1}\right) .
\end{aligned}
$$

\section{GMRES Method}

In the GMRES method, we let

$$
\mathbf{u}^{(n)}=\mathbf{u}^{(0)}+\mathbf{W}_{n-1} \mathbf{y}^{(n)}
$$

Multiplying by $\mathbf{A}$, we have

$$
\begin{gathered}
\mathbf{A} \mathbf{u}^{(n)}=\mathbf{A} \mathbf{u}^{(0)}+\left(\mathbf{A} \mathbf{W}_{n-1}\right) \mathbf{y}^{(n)}, \\
\mathbf{b}-\mathbf{A} \mathbf{u}^{(n)}=\mathbf{b}-\mathbf{A} \mathbf{u}^{(0)}-\left(\mathbf{A W}_{n-1}\right) \mathbf{y}^{(n)} .
\end{gathered}
$$

Then

$$
\mathbf{r}^{(n)}=\mathbf{r}^{(0)}-\left(\mathrm{AW}_{n-1}\right) \mathbf{y}^{(n)}
$$

For minimizing $\left\|\mathbf{r}^{(n)}\right\|$, we need to solve

$$
\min _{\mathbf{y} \in \mathbb{R}^{n}}\left\|\widetilde{\mathbf{H}}_{n+1} \mathbf{y}-\sigma_{0} \mathbf{e}_{1}\right\|
$$

by using Givens rotation, which is the GMRES method.

In Saad's book [4], there is a relation between the FOM method and the GMRES method. For the FOM method, we impose the Galerkin condition $\mathbf{r}^{(n)} \perp \mathbf{W}_{n-1}$ and we solve this linear system for $\mathbf{y}$

$$
\mathbf{H}_{n} \mathbf{y}=\sigma_{0} \mathbf{e}_{1}
$$

For the $(n+1)$ st iteration, we solve this linear system for $\mathbf{y}$

$$
\mathbf{H}_{n+1} \mathbf{y}=\sigma_{0} \mathbf{e}_{1} .
$$

Between the $n$th iteration and the $(n+1)$ st iteration of the FOM method, we obtain the solution of the this least squares problem

$$
\min _{\mathbf{y} \in \mathbb{R}^{n}}\left\|\widetilde{\mathbf{H}}_{n+1} \mathbf{y}-\sigma_{0} \mathbf{e}_{1}\right\|
$$

which is the same as in the GMRES method.

\section{Differences between MMINRES and MGMRES}

In this section, we assume that matrices $\mathbf{E}$ and EA are nonsingular symmetric matrices (but not necessarily positive definite). In $[6,7]$, we use a short term recurrence to generate orthonormal vectors $\left\{\mathbf{w}^{(0)}, \mathbf{w}^{(1)}, \mathbf{w}^{(2)}, \ldots, \mathbf{w}^{(n-3)}, \mathbf{w}^{(n-2)}, \mathbf{w}^{(n-1)}\right\}$ as follows:

$$
\begin{aligned}
& \mathbf{u}^{(0)}=\text { arbitrary, } \\
& \mathbf{r}^{(0)}=\mathbf{b}-\mathbf{A} \mathbf{u}^{(0)}, \\
& \widetilde{\mathbf{w}}^{(0)}=\mathbf{r}^{(0)}, \\
& \sigma_{0}=\sqrt{\left\langle\mathbf{E} \widetilde{\mathbf{w}}^{(0)}, \widetilde{\mathbf{w}}^{(0)}\right\rangle,} \\
& \mathbf{w}^{(0)}=\left(\frac{1}{\sigma_{0}}\right) \widetilde{\mathbf{w}}^{(0)},
\end{aligned}
$$

$$
\text { for } i=1,2, \ldots, n-1 \text {, }
$$

$$
\alpha_{i-1}=\frac{\left\langle\mathbf{E A w ^ { ( i - 1 ) }}, \mathbf{w}^{(i-1)}\right\rangle}{\left\langle\mathbf{E} \mathbf{w}^{(i-1)}, \mathbf{w}^{(i-1)}\right\rangle},
$$

$$
\beta_{i-1}=\frac{\left\langle\mathbf{E A w ^ { ( i - 1 ) }}, \mathbf{w}^{(i-2)}\right\rangle}{\left\langle\mathbf{E} \mathbf{w}^{(i-2)}, \mathbf{w}^{(i-2)}\right\rangle},
$$

$$
\widetilde{\mathbf{w}}^{(i)}=\mathbf{A} \mathbf{w}^{(i-1)}-\alpha_{i-1} \mathbf{w}^{(i-1)}-\beta_{i-1} \mathbf{w}^{(i-2)},
$$

$$
\begin{aligned}
& \sigma_{i}=\sqrt{\left|\left\langle\mathbf{E} \widetilde{\mathbf{w}}^{(i)}, \widetilde{\mathbf{w}}^{(i)}\right\rangle\right|}, \\
& \mathbf{w}^{(i)}=\left(\frac{1}{\sigma_{i}}\right) \widetilde{\mathbf{w}}^{(i)},
\end{aligned}
$$

end for.

We obtain these properties, for $(0 \leq i, j \leq n-1)$,

$$
\begin{aligned}
\left\langle\mathbf{E} \mathbf{w}^{(i)}, \mathbf{w}^{(j)}\right\rangle & =0, \quad \text { if } i \neq j, \\
\beta_{i} & =\sigma_{i},
\end{aligned}
$$

$$
\left(\mathbf{E W}_{n-1}\right)^{T} \mathbf{W}_{n-1}
$$

$$
=\left[\begin{array}{lllllll}
d_{0} & & & & & & \\
& d_{1} & & & & & \\
& & d_{2} & & & & \\
& & & \ddots & & & \\
& & & & d_{n-3} & & \\
& & & & & d_{n-2} & \\
& & & & & & d_{n-1}
\end{array}\right]_{n \times n}
$$

where

$$
d_{i}=\left\langle\mathbf{E} \mathbf{w}^{(i)}, \mathbf{w}^{(i)}\right\rangle= \begin{cases}1, & \text { if }\left\langle\mathbf{E} \widetilde{\mathbf{w}}^{(i)}, \widetilde{\mathbf{w}}^{(i)}\right\rangle>0, \\ -1, & \text { if }\left\langle\mathbf{E} \widetilde{\mathbf{w}}^{(i)}, \widetilde{\mathbf{w}}^{(i)}\right\rangle<0 .\end{cases}
$$


Consequently, we obtain these matrix equations

$$
\begin{aligned}
& \mathbf{A W}_{n-1}=\mathbf{W}_{n-1} \mathbf{T}_{n}+\sigma_{n} \mathbf{w}^{(n)} \mathbf{e}_{n}^{T} \\
& =\mathbf{W}_{n} \widehat{\mathbf{T}}_{n+1} \text {, } \\
& \mathbf{W}_{n-1}=\left[\mathbf{w}^{(0)}, \mathbf{w}^{(1)}, \mathbf{w}^{(2)}, \ldots, \mathbf{w}^{(n-3)}, \mathbf{w}^{(n-2)}, \mathbf{w}^{(n-1)}\right]_{n \times n}, \\
& \mathbf{e}_{n}^{T}=[0,0,0, \ldots, 0,0,0,1]_{1 \times n} \\
& \mathbf{T}_{n}=\left[\begin{array}{ccccccc}
\alpha_{0} & \beta_{1} & & & & & \\
\sigma_{1} & \alpha_{1} & \beta_{2} & & & & \\
& \sigma_{2} & \alpha_{2} & \beta_{3} & & & \\
& & \ddots & \ddots & \ddots & & \\
& & & \sigma_{n-3} & \alpha_{n-3} & \beta_{n-2} & \\
& & & & \sigma_{n-2} & \alpha_{n-2} & \beta_{n-1} \\
& & & & & \sigma_{n-1} & \alpha_{n-1}
\end{array}\right]_{n \times n} \\
& \widehat{\mathbf{T}}_{n+1} \\
& =\left[\begin{array}{ccccccc}
\alpha_{0} & \beta_{1} & & & & & \\
\sigma_{1} & \alpha_{1} & \beta_{2} & & & & \\
& \sigma_{2} & \alpha_{2} & \beta_{3} & & & \\
& & \ddots & \ddots & \ddots & & \\
& & & \sigma_{n-3} & \alpha_{n-3} & \beta_{n-2} & \\
& & & & \sigma_{n-2} & \alpha_{n-2} & \beta_{n-1} \\
& & & & & \sigma_{n-1} & \alpha_{n-1} \\
& & & & & & \sigma_{n}
\end{array}\right]_{(n+1) \times n}
\end{aligned}
$$

From (134) and (132), we obtain

$$
\begin{aligned}
& \left(\mathbf{E W}_{n-1}\right)^{T}\left(\mathbf{A} \mathbf{W}_{n-1}\right) \\
& =\left(\mathbf{E W}_{n-1}\right)^{T}\left(\mathbf{W}_{n-1} \mathbf{T}_{n}+\sigma_{n} \mathbf{w}^{(n)} \mathbf{e}_{n}^{T}\right) \\
& =\left(\mathbf{E W}_{n-1}\right)^{T} \mathbf{W}_{n-1} \mathbf{T}_{n}+\left(\mathbf{E} \mathbf{W}_{n-1}\right)^{T} \sigma_{n} \mathbf{w}^{(n)} \mathbf{e}_{n}^{T} \\
& =\left(\mathbf{E W}_{n-1}\right)^{T} \mathbf{W}_{n-1} \mathbf{T}_{n}+\sigma_{n}\left\langle\mathbf{E} \mathbf{W}_{n-1}, \mathbf{w}^{(n)}\right\rangle \mathbf{e}_{n}^{T} \\
& =\mathbf{D}_{n} \mathbf{T}_{n}+\mathbf{0}=\mathbf{D}_{n} \mathbf{T}_{n} .
\end{aligned}
$$

Assume that $\mathbf{x}^{(n)}-\mathbf{x}^{(0)} \in \mathbf{W}_{n-1}$; then we have

$$
\begin{aligned}
& \mathbf{x}^{(n)}=\mathbf{x}^{(0)}+\mathbf{W}_{n-1} \mathbf{y}^{(n)} \\
& \mathbf{r}^{(n)}=\mathbf{r}^{(0)}-\left(\mathbf{A W}_{n-1}\right) \mathbf{y}^{(n)} .
\end{aligned}
$$

From the Galerkin condition $\mathbf{E r}^{(n)} \perp \mathbf{W}_{n-1}$, we have

$$
\left(\mathbf{E r}^{(n)}\right)^{T} \mathbf{W}_{n-1}=\mathbf{0}
$$

From (141) and since $\mathbf{E}$ is symmetric, we obtain

$$
\begin{aligned}
\mathbf{0} & =\left(\mathbf{E} \mathbf{r}^{(n)}\right)^{T} \mathbf{W}_{n-1}=\left(\mathbf{r}^{(n)}\right)^{T} \mathbf{E}^{T} \mathbf{W}_{n-1} \\
& =\left(\mathbf{r}^{(n)}\right)^{T} \mathbf{E W}_{n-1}=\left(\mathbf{E} \mathbf{W}_{n-1}\right)^{T} \mathbf{r}^{(n)} .
\end{aligned}
$$

Then by (142), we have

$$
\left(\mathbf{E W}_{n-1}\right)^{T} \mathbf{r}^{(n)}=\mathbf{0}
$$

From (139), (140), and (143), we obtain this linear system

$$
\begin{aligned}
\left(\mathbf{D}_{n} \mathbf{T}_{n}\right) \mathbf{y}^{(n)} & =\left(\mathbf{E} \mathbf{W}_{n-1}\right)^{T}\left(\mathbf{A} \mathbf{W}_{n-1}\right) \mathbf{y}^{(n)} \\
& =\left(\mathbf{E} \mathbf{W}_{n-1}\right)^{T}\left(\mathbf{r}^{(0)}-\mathbf{r}^{(n)}\right) \\
& =\left(\mathbf{E} \mathbf{W}_{n-1}\right)^{T} \mathbf{r}^{(0)} \\
& =\sigma_{0}\left(\mathbf{E W}_{n-1}\right)^{T} \mathbf{w}^{(0)} \\
& =\sigma_{0}\left\langle\mathbf{E} \mathbf{W}_{n-1}, \mathbf{w}^{(0)}\right\rangle \\
& =\sigma_{0} \mathbf{e}_{n}
\end{aligned}
$$

using (127), where $\sigma_{0}=d_{0} \sqrt{\left|\left\langle\mathbf{E r}^{(0)}, \mathbf{r}^{(0)}\right\rangle\right|}$ and $\sigma_{0} \mathbf{w}^{(0)}=\mathbf{r}^{(0)}$. By (132) and (137), we have

$$
\mathbf{D}_{n} \mathbf{T}_{n}=\left[\begin{array}{ccccccc}
d_{0} \alpha_{0} & d_{0} \beta_{1} & & & & \\
d_{1} \sigma_{1} & d_{1} \alpha_{1} & d_{1} \beta_{2} & & & & \\
& d_{2} \sigma_{2} & d_{2} \alpha_{2} & d_{2} \beta_{3} & & \\
& & \ddots & \ddots & \ddots & & \\
& & & d_{n-3} \sigma_{n-3} & d_{n-3} \sigma_{n-3} & d_{n-3} \beta_{n-2} & \\
& & & d_{n-2} \sigma_{n-2} & d_{n-2} \alpha_{n-2} & d_{n-2} \beta_{n-1} \\
& & & & & d_{n-1} \sigma_{n-1} & d_{n-1} \alpha_{n-1}
\end{array}\right]_{n \times n}
$$


Notice that $\mathbf{D}_{n} \mathbf{T}_{n}$ is symmetric since

$$
\begin{aligned}
d_{i-1} \beta_{i}= & \frac{d_{i-1}\left\langle\mathbf{E} \mathbf{A} \mathbf{w}^{(i)}, \mathbf{w}^{(i-1)}\right\rangle}{\left\langle\mathbf{E} \mathbf{w}^{(i-1)}, \mathbf{w}^{(i-1)}\right\rangle} \\
= & \frac{d_{i-1}\left\langle\mathbf{w}^{(i)}, \mathbf{E} \mathbf{A} \mathbf{w}^{(i-1)}\right\rangle}{\left\langle\mathbf{E} \mathbf{w}^{(i-1)}, \mathbf{w}^{(i-1)}\right\rangle} \\
= & \frac{d_{i-1}\left\langle\mathbf{w}^{(i)}, \mathbf{E}\left(\sigma_{i} \mathbf{w}^{(i)}+\alpha_{i-1} \mathbf{w}^{(i-1)}+\beta_{i-1} \mathbf{w}^{(i-2)}\right)\right\rangle}{\left\langle\mathbf{E} \mathbf{w}^{(i-1)}, \mathbf{w}^{(i-1)}\right\rangle} \\
= & \left(d _ { i - 1 } \left[\sigma_{i}\left\langle\mathbf{E} \mathbf{w}^{(i)}, \mathbf{w}^{(i)}\right\rangle+\alpha_{i-1}\left\langle\mathbf{E} \mathbf{w}^{(i)}, \mathbf{w}^{(i-1)}\right\rangle\right.\right. \\
& \left.\left.+\beta_{i-1}\left\langle\mathbf{E} \mathbf{w}^{(i)}, \mathbf{w}^{(i-2)}\right\rangle\right]\right) \\
& \times\left(\left\langle\mathbf{E} \mathbf{w}^{(i-1)}, \mathbf{w}^{(i-1)}\right\rangle\right)^{-1} \\
= & \frac{d_{i-1} \sigma_{i}\left\langle\mathbf{E} \mathbf{w}^{(i)}, \mathbf{w}^{(i)}\right\rangle}{\left\langle\mathbf{E} \mathbf{w}^{(i-1)}, \mathbf{w}^{(i-1)}\right\rangle} \\
d_{i-1} \sigma_{i} d_{i} & d_{i} \sigma_{i} .
\end{aligned}
$$

Here we use (128), EA is symmetric, (129), (130), E is symmetric, (131), and (133).

Let $\overline{\mathbf{x}}_{\text {sym }}^{(n)}$ be the estimated solution corresponding to the Galerkin condition. Then

$$
\overline{\mathbf{x}}_{\mathrm{sym}}^{(n)}=\mathbf{x}^{(0)}+\mathbf{W}_{n-1} \overline{\mathbf{y}}^{(n)},
$$

where $\overline{\mathbf{y}}^{(n)}$ satisfies this linear system

$$
\left(\mathbf{D}_{n} \mathbf{T}_{n}\right) \overline{\mathbf{y}}^{(n)}=\sigma_{0} \mathbf{e}_{n}
$$

Let $\mathbf{x}_{\min }^{(n)}$ be the estimated solution corresponding to the least squares problem. Then

$$
\mathbf{x}_{\min }^{(n)}=\mathbf{x}^{(0)}+\mathbf{W}_{n-1} \mathbf{y}^{(n)}
$$

where $\mathbf{y}^{(n)}$ satisfies this expression

$$
\min _{\mathbf{y}^{(n)} \in \mathbb{R}^{n}}\left\|\left(\mathbf{D}_{n+1} \widehat{\mathbf{T}}_{n+1}\right) \mathbf{y}^{(n)}-\sigma_{0} \mathbf{e}_{n+1}\right\|
$$

We call $\mathbf{x}_{\min }^{(n)}$ the $n$th iteration of the MMINRES method.

Notice that if to matrix $\mathbf{D}_{n+1} \mathbf{T}_{n+1}$ we add this bottom row

$$
\left[0,0,0, \ldots 0,0,0, d_{n} \sigma_{n}\right]_{1 \times n}
$$

and add this far-right column

$$
\left[0,0,0, \ldots 0,0,0, d_{n-1} \beta_{n}, d_{n} \alpha_{n}\right]_{1 \times n+1}^{T} \text {, }
$$

see (132) and (138), then we obtain the matrix $\mathbf{D}_{n+1} \widehat{\mathbf{T}}_{n+1}$.
We define these Given rotation matrices

$$
\mathbf{Q}_{n-1} \equiv \mathbf{Q}_{n-1, n} \mathbf{Q}_{n-2, n-1} \mathbf{Q}_{n-3, n-2} \cdots \mathbf{Q}_{3,4} \mathbf{Q}_{2,3} \mathbf{Q}_{1,2},
$$

where $\mathbf{Q}_{i, i+1}$ is given by (23). Multiplying matrix $\mathbf{Q}_{n-1}$ times both sides of linear system (148), we obtain this linear system

$$
\left(\mathbf{Q}_{n-1} \mathbf{D}_{n} \mathbf{T}_{n}\right) \overline{\mathbf{y}}^{(n)}=\mathbf{Q}_{n-1} \sigma_{0} \mathbf{e}_{n}
$$

where, from (137), the coefficient matrix is

$$
\mathbf{Q}_{n-1} \mathbf{D}_{n} \mathbf{T}_{n}
$$

$$
\begin{aligned}
& {\left[\begin{array}{cccccc|c}
\gamma_{0} & \delta_{1} & \varepsilon_{2} & & & & \\
& \gamma_{1} & \delta_{2} & \varepsilon_{3} & & & \\
& & \gamma_{2} & \delta_{3} & \varepsilon_{4} & & \\
& & & \ddots & \ddots & \ddots & \\
& & & & \gamma_{n-3} & \delta_{n-2} & \varepsilon_{n-1} \\
& & & & & \gamma_{n-2} & \delta_{n-1} \\
& & & & & & \bar{\gamma}_{n-1}
\end{array}\right]_{n \times n} } \\
\equiv & \overline{\mathbf{R}}_{n},
\end{aligned}
$$

and the right-hand side vector is

$$
\begin{aligned}
\mathbf{Q}_{n-1} \sigma_{0} \mathbf{e}_{n} & =\left[\zeta_{0}, \zeta_{1}, \zeta_{2}, \ldots, \zeta_{n-3}, \zeta_{n-2}, \bar{\zeta}_{n-1}\right]_{1 \times n}^{T} \\
& \equiv \mathbf{z}^{(n)} .
\end{aligned}
$$

Note that $\bar{\gamma}_{n-1}$ is not always nonzero, so that $\overline{\mathbf{R}}_{n}$ might be singular, but here, we assume that $\overline{\mathbf{R}}_{n}$ is nonsingular. Then we define this matrix factorization

$$
\mathbf{W}_{n-1}=\overline{\mathbf{P}}_{n} \overline{\mathbf{R}}_{n}
$$

where

$$
\overline{\mathbf{P}}_{n} \equiv\left[\mathbf{p}^{(0)}, \mathbf{p}^{(1)}, \mathbf{p}^{(2)}, \ldots, \mathbf{p}^{(n-3)}, \mathbf{p}^{(n-2)}, \overline{\mathbf{p}}^{(n-1)}\right]_{n \times n}
$$

Moreover, we define the right-hand side vector in (156) as

$$
\overline{\mathbf{z}}^{(n)}=\overline{\mathbf{R}}_{n} \overline{\mathbf{y}}^{(n)} .
$$

From (147), we have, using (157) and (159),

$$
\begin{aligned}
\overline{\mathbf{x}}_{\mathrm{sym}}^{(n)} & =\mathbf{x}^{(0)}+\mathbf{W}_{n-1} \overline{\mathbf{y}}^{(n)} \\
& =\mathbf{x}^{(0)}+\overline{\mathbf{P}}_{n} \overline{\mathbf{R}}_{n} \overline{\mathbf{y}}^{(n)} \\
& =\mathbf{x}^{(0)}+\overline{\mathbf{P}}_{n} \overline{\mathbf{z}}^{(n)}
\end{aligned}
$$

For the next iterate $\overline{\mathbf{x}}_{\text {sym }}^{(n+1)}$, we need to solve this linear system

$$
\left(\mathbf{D}_{n+1} \mathbf{T}_{n+1}\right) \overline{\mathbf{y}}^{(n+1)}=\sigma_{0} \mathbf{e}_{n+1},
$$


where, by (132) and (137), the coefficient matrix is

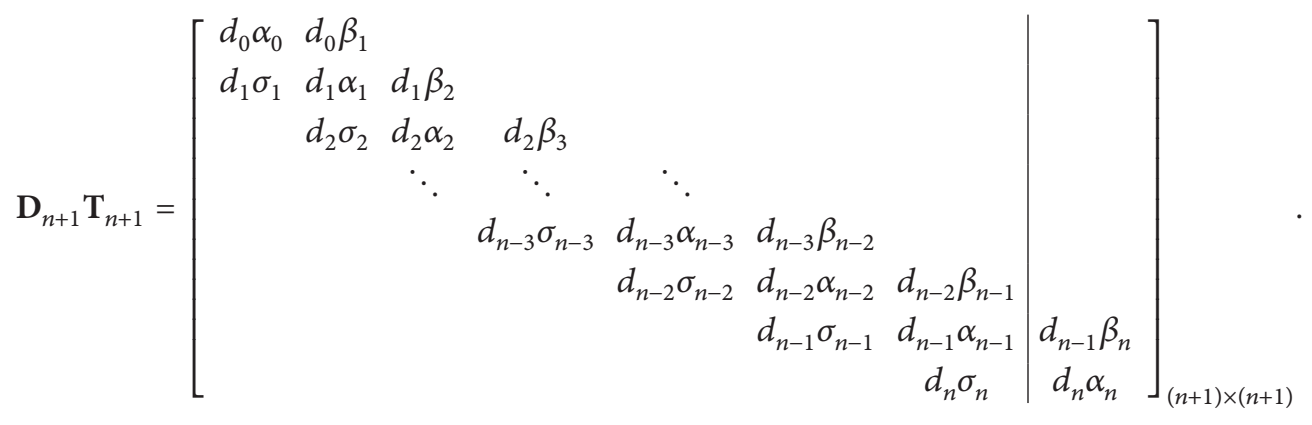

Multiplying $\mathbf{Q}_{n-1}$ times both sides of linear system (161), we obtain this coefficient matrix

$$
\mathbf{Q}_{n-1}\left(\mathbf{D}_{n+1} \mathbf{T}_{n+1}\right)=\left[\begin{array}{ccccccc|c}
\gamma_{0} & \delta_{1} & \varepsilon_{2} & & & & \\
& \gamma_{1} & \delta_{2} & \varepsilon_{3} & & & & \\
& & \gamma_{2} & \delta_{3} & \varepsilon_{4} & & \\
& & & \ddots & \ddots & \ddots & & \\
& & & & \gamma_{n-3} & \delta_{n-2} & \varepsilon_{n-1} & \\
& & & & & \gamma_{n-2} & \delta_{n-1} & \varepsilon_{n} \\
& & & & & & \bar{\gamma}_{n-1} & \psi \\
& & & & & & d_{n} \sigma_{n} & d_{n} \alpha_{n}
\end{array}\right]_{(n+1) \times(n+1)}
$$

The last columns in matrices (162) and (163) are related as follows:

$$
\begin{gathered}
\mathbf{Q}_{n-1, n}\left[0,0,0, \ldots 0,0,0,0, d_{n-1} \beta_{n}, d_{n} \alpha_{n}\right]_{(n+1) \times 1}^{T} \\
=\left[0,0,0, \ldots 0,0,0, \varepsilon_{n}, \psi, d_{n} \alpha_{n}\right]_{(n+1) \times 1}^{T} .
\end{gathered}
$$

To eliminate $d_{n} \sigma_{n}$, we compute the $n$th Givens rotation matrix $\mathbf{Q}_{n, n+1}$ with

$$
\gamma_{n-1}=\sqrt{\bar{\gamma}_{n-1}^{2}+\left(d_{n} \sigma_{n}\right)^{2}}, \quad c_{n}=\frac{\bar{\gamma}_{n-1}}{\gamma_{n-1}}, \quad s_{n}=-\frac{d_{n} \sigma_{n}}{\gamma_{n-1}} .
$$

Recall, by (23), that $c_{i}^{2}+s_{i}^{2}=1$. Next, we multiply matrix $\mathbf{Q}_{n, n+1}$ times the coefficient matrix $\mathbf{Q}_{n-1}\left(\mathbf{D}_{n+1} \mathbf{T}_{n+1}\right)$, in (163),

$$
\mathbf{Q}_{n, n+1} \mathbf{Q}_{n-1}\left(\mathbf{D}_{n+1} \mathbf{T}_{n+1}\right)=\left[\begin{array}{ccccccc|c}
\gamma_{0} & \delta_{1} & \varepsilon_{2} & & & & \\
& \gamma_{1} & \delta_{2} & \varepsilon_{3} & & & \\
& & \gamma_{2} & \delta_{3} & \varepsilon_{4} & & \\
& & & \ddots & \ddots & \ddots & \\
& & & & \gamma_{n-3} & \delta_{n-2} & \varepsilon_{n-1} & \\
& & & & & \gamma_{n-2} & \delta_{n-1} & \varepsilon_{n} \\
& & & & & & \gamma_{n-1} & \delta_{n} \\
& & & & & & & \bar{\gamma}_{n}
\end{array}\right]_{(n+1) \times(n+1)} \equiv \overline{\mathbf{R}}_{n+1}
$$

Then multiplying matrix $\mathbf{Q}_{n, n+1}$ times the right-hand side vector $\mathbf{Q}_{n-1} \sigma_{0} \mathbf{e}_{n+1}$, we obtain

$$
\begin{aligned}
\mathbf{Q}_{n, n+1}\left(\mathbf{Q}_{n-1} \sigma_{0} \mathbf{e}_{n+1}\right) & =\left[\zeta_{0}, \zeta_{1}, \zeta_{2}, \ldots, \zeta_{n-2}, \zeta_{n-1}, \bar{\zeta}_{n}\right]_{(n+1) \times 1}^{T} \\
& \equiv \overline{\mathbf{z}}_{n+1} .
\end{aligned}
$$

Looking at the last columns in (163) and (166), we see that

$$
\begin{aligned}
\mathbf{Q}_{n, n+1} & {\left[0,0,0, \ldots 0,0,0, \varepsilon_{n}, \psi, d_{n} \alpha_{n}\right]_{(n+1) \times 1}^{T} } \\
& =\left[0,0,0, \ldots, 0,0,0, \varepsilon_{n}, \delta_{n}, \bar{\gamma}_{n}\right]_{(n+1) \times 1}^{T},
\end{aligned}
$$


where

$$
\zeta_{n-1}=c_{n} \bar{\zeta}_{n-1}, \quad \bar{\zeta}_{n}=s_{n} \bar{\zeta}_{n-1} .
$$

Recall, by (23), $c_{n}^{2}+s_{n}^{2}=1$.

Before forming the iterate $\overline{\mathbf{x}}_{\text {sym }}^{(n+1)}$, we consider this least square problem (150)

$$
\min _{\mathbf{y}^{(n)} \in \mathbb{R}^{n}}\left\|\left(\mathbf{D}_{n+1} \widehat{\mathbf{T}}_{n+1}\right) \mathbf{y}^{(n)}-\sigma_{0} \mathbf{e}_{n+1}\right\| .
$$

We have

$$
\begin{aligned}
& \left\|\mathbf{Q}_{n, n+1} \mathbf{Q}_{n-1}\left(\mathbf{D}_{n+1} \widehat{\mathbf{T}}_{n+1} \mathbf{y}^{(n)}-\sigma_{0} \mathbf{e}_{n+1}\right)\right\| \\
& \left.=\| \begin{array}{c}
\zeta_{0} \\
\zeta_{1} \\
\zeta_{2} \\
\vdots \\
{\left[\begin{array}{l}
\mathbf{R}_{n} \\
0,0,0, \ldots, 0,0,0
\end{array}\right]_{(n+1) \times n} \mathbf{y}^{(n)}-\left[\begin{array}{c}
\zeta_{n-2} \\
\zeta_{n-1} \\
\bar{\zeta}_{n}
\end{array}\right]_{(n+1) \times 1}}
\end{array}\right],
\end{aligned}
$$

where

$$
\mathbf{R}_{n} \equiv\left[\begin{array}{ccccccc|c}
\gamma_{0} & \delta_{1} & \varepsilon_{2} & & & & \\
& \gamma_{1} & \delta_{2} & \varepsilon_{3} & & & \\
& & \gamma_{2} & \delta_{3} & \varepsilon_{4} & & \\
& & & \ddots & \ddots & \ddots & & \\
& & & & \gamma_{n-4} & \delta_{n-3} & \varepsilon_{n-2} & \\
& & & & & \gamma_{n-3} & \delta_{n-2} & \varepsilon_{n-1} \\
& & & & & & \gamma_{n-2} & \delta_{n-1} \\
& & & & & & & \gamma_{n-1}
\end{array}\right]_{n \times n}
$$

Next define

$$
\mathbf{z}^{(n)} \equiv\left[\zeta_{0}, \zeta_{1}, \zeta_{2}, \ldots, \zeta_{n-3}, \zeta_{n-2}, \zeta_{n-1}\right]_{1 \times n}^{T} .
$$

Hence, $\mathbf{y}^{(n)}$ is the solution of this linear system

$$
\mathbf{R}_{n} \mathbf{y}^{(n)}=\mathbf{z}^{(n)},
$$

which minimizes the expression

$$
\min _{\mathbf{y}^{(n)} \in \mathbb{R}^{n}}\left\|\left(\mathbf{D}_{n+1} \widehat{\mathbf{T}}_{n+1}\right) \mathbf{y}^{(n)}-\sigma_{0} \mathbf{e}_{n+1}\right\|,
$$

and then

$$
\bar{\zeta}_{n}=\left\|\left(\mathbf{D}_{n+1} \widehat{\mathbf{T}}_{n+1}\right) \mathbf{y}^{(n)}-\sigma_{0} \mathbf{e}_{n+1}\right\| .
$$

If $\sigma_{n} \neq 0$, then $\gamma_{n} \neq 0$ and $\mathbf{R}_{n}$ is nonsingular, and we can solve linear system (174) for $\mathbf{y}^{(n)}$. We discuss the case $\sigma_{n}=0$ later.

By (149), let

$$
\begin{aligned}
\mathbf{x}_{\min }^{(n)} & =\mathbf{x}^{(0)}+\mathbf{W}_{n-1} \mathbf{y}^{(n)} \\
& =\mathbf{x}^{(0)}+\left(\mathbf{W}_{n-1} \mathbf{R}_{n}^{-1}\right) \mathbf{z}^{(n)},
\end{aligned}
$$

using (174). Here

$$
\begin{gathered}
\mathbf{W}_{n-1}=\mathbf{P}_{n} \mathbf{R}_{n}, \\
\mathbf{P}_{n-1}=\left[\mathbf{p}^{(0)}, \mathbf{p}^{(1)}, \mathbf{p}^{(2)}, \ldots, \mathbf{p}^{(n-3)}, \mathbf{p}^{(n-2)}, \mathbf{p}^{(n-1)}\right]_{n \times n} .
\end{gathered}
$$

From (177) and (179), we have

$$
\begin{aligned}
\mathbf{x}_{\min }^{(n)} & =\mathbf{x}^{(0)}+\mathbf{P}_{n} \mathbf{z}^{(n)} \\
& =\mathbf{x}_{\min }^{(n-1)}+\zeta_{n-1} \mathbf{p}^{(n-1)},
\end{aligned}
$$

where, by (172),

$$
\mathbf{p}^{(n-1)}=\left(\frac{1}{\gamma_{n-1}}\right)\left[\mathbf{w}^{(n-1)}-\delta_{n-1} \mathbf{p}^{(n-2)}-\varepsilon_{n-1} \mathbf{p}^{(n-3)}\right] .
$$

From (147), we obtain, by (159), (157), and (180),

$$
\begin{aligned}
\overline{\mathbf{x}}_{\text {sym }}^{(n+1)} & =\mathbf{x}^{(0)}+\mathbf{W}_{n} \overline{\mathbf{y}}^{(n+1)} \\
& =\mathbf{x}^{(0)}+\mathbf{W}_{n}\left(\mathbf{R}_{n+1}^{-1} \mathbf{z}^{(n+1)}\right) \\
& =\mathbf{x}^{(0)}+\left(\mathbf{W}_{n} \overline{\mathbf{R}}_{n+1}^{-1}\right) \overline{\mathbf{z}}^{(n+1)} \\
& =\mathbf{x}^{(0)}+\overline{\mathbf{P}}_{n+1} \overline{\mathbf{z}}^{(n+1)} \\
& =\mathbf{x}^{(0)}+\mathbf{P}_{n-1} \mathbf{z}^{(n)}+\bar{\zeta}_{n} \overline{\mathbf{p}}^{(n)} \\
& =\mathbf{x}_{\min }^{(n)}+\bar{\zeta}_{n} \overline{\mathbf{p}}^{(n)} .
\end{aligned}
$$

We note that $\overline{\mathbf{x}}_{\text {sym }}^{(n)}$ is the estimated solution satisfying the Galerkin condition, while

$$
\mathbf{x}_{\min }^{(n)}=\mathbf{x}^{(0)}+\mathbf{W}_{n-1} \mathbf{y}^{(n)},
$$

where $\mathbf{y}^{(n)}$ minimizes this expression

$$
\min _{\mathbf{y}^{(n)} \in \mathbb{R}^{n}}\left\|\left(\mathbf{D}_{n+1} \widehat{\mathbf{T}}_{n+1}\right) \mathbf{y}^{(n)}-\sigma_{0} \mathbf{e}_{n+1}\right\| .
$$

Proposition 1. If $\sigma_{n}=0$ and $\widetilde{\mathbf{w}}^{(n)}=\mathbf{0}$, then $\overline{\mathbf{x}}_{\text {sym }}^{(n)}$ is the exact solution of $\mathbf{A u}=\mathbf{b}$. Moreover, one obtains $\mathbf{x}_{\min }^{(n)}=\overline{\mathbf{x}}_{\text {sym }}^{(n)}$. 
Proof. We consider the residual vector $\overline{\mathbf{r}}^{(n)}$ corresponding to the iterate $\overline{\mathbf{x}}_{\text {sym }}^{(n)}$. Then, by (147), we have

$$
\begin{aligned}
\overline{\mathbf{r}}^{(n)} & =\mathbf{b}-\mathbf{A} \overline{\mathbf{x}}_{\mathrm{sym}}^{(n)} \\
& =\mathbf{b}-\mathbf{A}\left(\mathbf{x}^{(0)}+\mathbf{W}_{n-1} \overline{\mathbf{y}}^{(n)}\right) \\
& =\left(\mathbf{b}-\mathbf{A} \mathbf{x}^{(0)}\right)-\left(\mathbf{A} \mathbf{W}_{n-1}\right) \overline{\mathbf{y}}^{(n)} \\
& =\mathbf{r}^{(0)}-\left(\mathbf{A} \mathbf{W}_{n-1}\right) \overline{\mathbf{y}}^{(n)} .
\end{aligned}
$$

Here $\overline{\mathbf{y}}^{(n)}$ satisfies (148) and we have

$$
\begin{gathered}
\left(\mathbf{D}_{n} \mathbf{T}_{n}\right) \overline{\mathbf{y}}^{(n)}=\sigma_{0} \mathbf{e}_{n}, \\
\mathbf{T}_{n} \overline{\mathbf{y}}^{(n)}=\mathbf{D}_{n}^{-1} \sigma_{0} \mathbf{e}_{n} .
\end{gathered}
$$

Hence, starting from (185) and by using (134) and (187), we have

$$
\begin{aligned}
\overline{\mathbf{r}}^{(n)} & =\mathbf{r}^{(0)}-\left(\mathbf{A} \mathbf{W}_{n-1}\right) \overline{\mathbf{y}}^{(n)} \\
& =\mathbf{r}^{(0)}-\left(\mathbf{W}_{n-1} \mathbf{T}_{n}+\sigma_{n} \mathbf{w}^{(n)} \mathbf{e}_{n}^{\mathbf{T}}\right) \overline{\mathbf{y}}^{(n)} \\
& =\mathbf{r}^{(0)}-\mathbf{W}_{n-1}\left(\mathbf{T}_{n} \overline{\mathbf{y}}^{(n)}\right)-\sigma_{n} \mathbf{w}^{(n)} \mathbf{e}_{n}^{T} \overline{\mathbf{y}}^{(n)} \\
& =\mathbf{r}^{(0)}-\mathbf{W}_{n-1}\left(\mathbf{D}_{n}^{-1} \sigma_{0} \mathbf{e}_{n}\right)-\sigma_{n} \mathbf{w}^{(n)} \mathbf{e}_{n}^{T} \overline{\mathbf{y}}^{(n)} \\
& =\sigma_{0} \mathbf{w}^{(0)}-\sigma_{0} \mathbf{w}^{(0)}-\sigma_{n} \mathbf{w}^{(n)} \mathbf{e}_{n}^{T} \overline{\mathbf{y}}^{(n)} \\
& =\mathbf{0}-\sigma_{n} \mathbf{w}^{(n)} \mathbf{e}_{n}^{T} \overline{\mathbf{y}}^{(n)} \\
& =-\sigma_{n} \bar{\eta}_{n} \mathbf{w}^{(n)},
\end{aligned}
$$

since $\mathbf{r}^{(0)}=\sigma_{0} \mathbf{w}^{(0)}$. Here $\bar{\eta}_{n}$ is the $n$th component of the vector $\overline{\mathbf{y}}^{(n)}$.

If $\sigma_{n}=0$, then $\overline{\mathbf{r}}^{(n)}=\mathbf{0}$. Thus, we obtain $\overline{\mathbf{x}}_{\mathrm{sym}}^{(n)}$, which is the exact solution. It follows from (165) that if $\sigma_{n}=0$, then

$$
\begin{aligned}
& c_{n}=\frac{\bar{\gamma}_{n-1}}{\left|\bar{\gamma}_{n-1}\right|}= \begin{cases}1, & \bar{\gamma}_{n-1}>0, \\
-1, & \bar{\gamma}_{n-1}<0,\end{cases} \\
& s_{n}=0 .
\end{aligned}
$$

Since

$$
\begin{array}{ll}
\overline{\mathbf{x}}_{\text {sym }}^{(n)}=\mathbf{x}^{(0)}+\mathbf{W}_{n-1} \overline{\mathbf{y}}^{(n)}, & \overline{\mathbf{R}}_{n} \overline{\mathbf{y}}^{(n)}=\overline{\mathbf{z}}^{(n)}, \\
\mathbf{x}_{\min }^{(n)}=\mathbf{x}^{(0)}+\mathbf{W}_{n-1} \mathbf{y}^{(n)}, & \mathbf{R}_{n} \mathbf{y}^{(n)}=\mathbf{z}^{(n)},
\end{array}
$$

the only difference between $\overline{\mathbf{R}}_{n}$ and $\mathbf{R}_{n}$ is the $(k, k)$-entries, which are $\bar{\gamma}_{n-1}$ and $\gamma_{n-1}$, respectively. Let the $n$th components of $\overline{\mathbf{y}}^{(n)}$ and $\mathbf{y}^{(n)}$ be $\bar{\eta}_{n}$ and $\eta_{n}$, respectively. It follows that both matrices $\overline{\mathbf{R}}_{n}$ and $\mathbf{R}_{n}$ are triangular, where $\eta_{n}=\zeta_{n-1} / \gamma_{n-1}$ and $\bar{\eta}_{n}=\bar{\zeta}_{n-1} / \bar{\gamma}_{n-1}$. By (169), we have

$$
\eta_{n}=\frac{c_{n} \bar{\zeta}_{n-1}}{\left|\bar{\gamma}_{n-1}\right|}=\frac{\bar{\zeta}_{n-1}}{\bar{\gamma}_{n-1}}=\bar{\eta}_{n} .
$$

Hence, we have

$$
\mathbf{y}^{(n)}=\overline{\mathbf{y}}^{(n)}, \quad \mathbf{x}_{\min }^{(n)}=\overline{\mathbf{x}}_{\mathrm{sym}}^{(n)} .
$$

\section{Final Notes}

In the MGMRES method, we solve this linear system

$$
\left(\widetilde{\mathbf{T}}_{n+1}^{T} \mathbf{D}_{n+1} \mathbf{T}_{n+1}\right) \mathbf{y}^{(n+1)}=\sigma_{0} \mathbf{e}_{1},
$$

while in the MMINRES method, we solve this linear system

$$
\left(\widetilde{\mathbf{T}}_{n+1}^{T} \widetilde{\mathbf{T}}_{n+1}\right) \mathbf{y}^{(n+1)}=\mathbf{D}_{n+1} \sigma_{0} \mathbf{e}_{1}
$$

Clearly, the MMINRES method and the MGMRES method are the same when the diagonal matrix $\mathbf{D}_{n+1}$ is the identity matrix $\mathbf{I}_{n+1}$.

See also Kincaid et al. [8].

\section{Conflict of Interests}

The authors declare that there is no conflict of interests regarding the publication of this paper.

\section{References}

[1] C. Lanczos, "An iteration method for the solution of the eigenvalue problem of linear differential and integral operators," Journal of Research of the National Bureau of Standards, vol. 45, pp. 255-282, 1950.

[2] G. H. Golub and C. F. Van Loan, Matrix Computations, Johns Hopkins University Press, 3rd edition, 1996.

[3] C. C. Paige and M. A. Saunders, "Solutions of sparse indefinite systems of linear equations," SIAM Journal on Numerical Analysis, vol. 12, no. 4, pp. 617-629, 1975.

[4] Y. Saad, Iterative Methods for Sparse Linear Systems, SIAM, 2nd edition, 2003.

[5] Y. Saad and M. H. Schultz, "GMRES: a generalized minimal residual algorithm for solving nonsymmetric linear systems," Society for Industrial and Applied Mathematics, vol. 7, no. 3, pp. 856-869, 1986.

[6] Y. Li, J. Chen, and D. R. Kincaid, "Some generalizations and modifications of iterative methods for solving large sparse symmetric indefinite linear systems," Abstract and Applied Analysis, vol. 2014, Article ID 237808, 11 pages, 2014.

[7] Y. Li, The modified, MINRES methods for solving large sparse non-symmetric linear systems [M.S. thesis], Department of Mathematics, National Kaohsiung Normal University, Kaohsiung, Taiwan, 2007.

[8] D. R. Kincaid, D. M. Young, and J.-Y. Chen, "Overview of MGMRES and LAN/MGMRES methods for solving nonsymmetric linear systems," Taiwanese Journal of Mathematics, vol. 4, no. 3, pp. 385-396, 2000. 


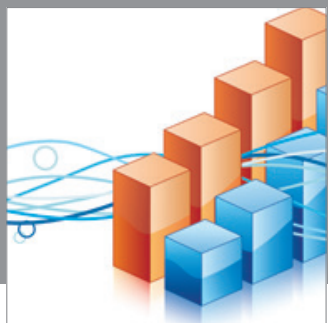

Advances in

Operations Research

mansans

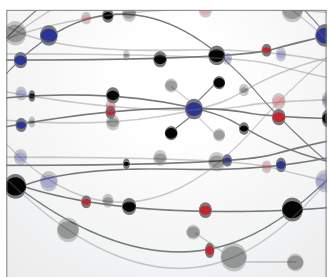

The Scientific World Journal
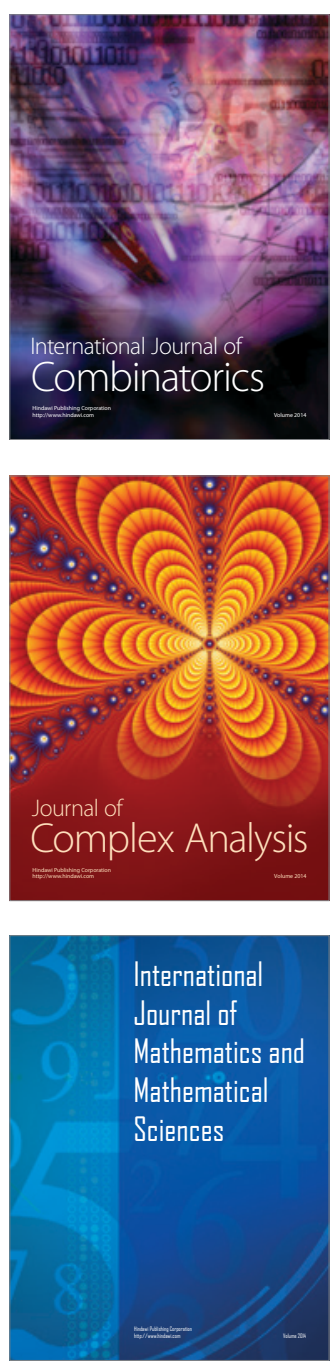
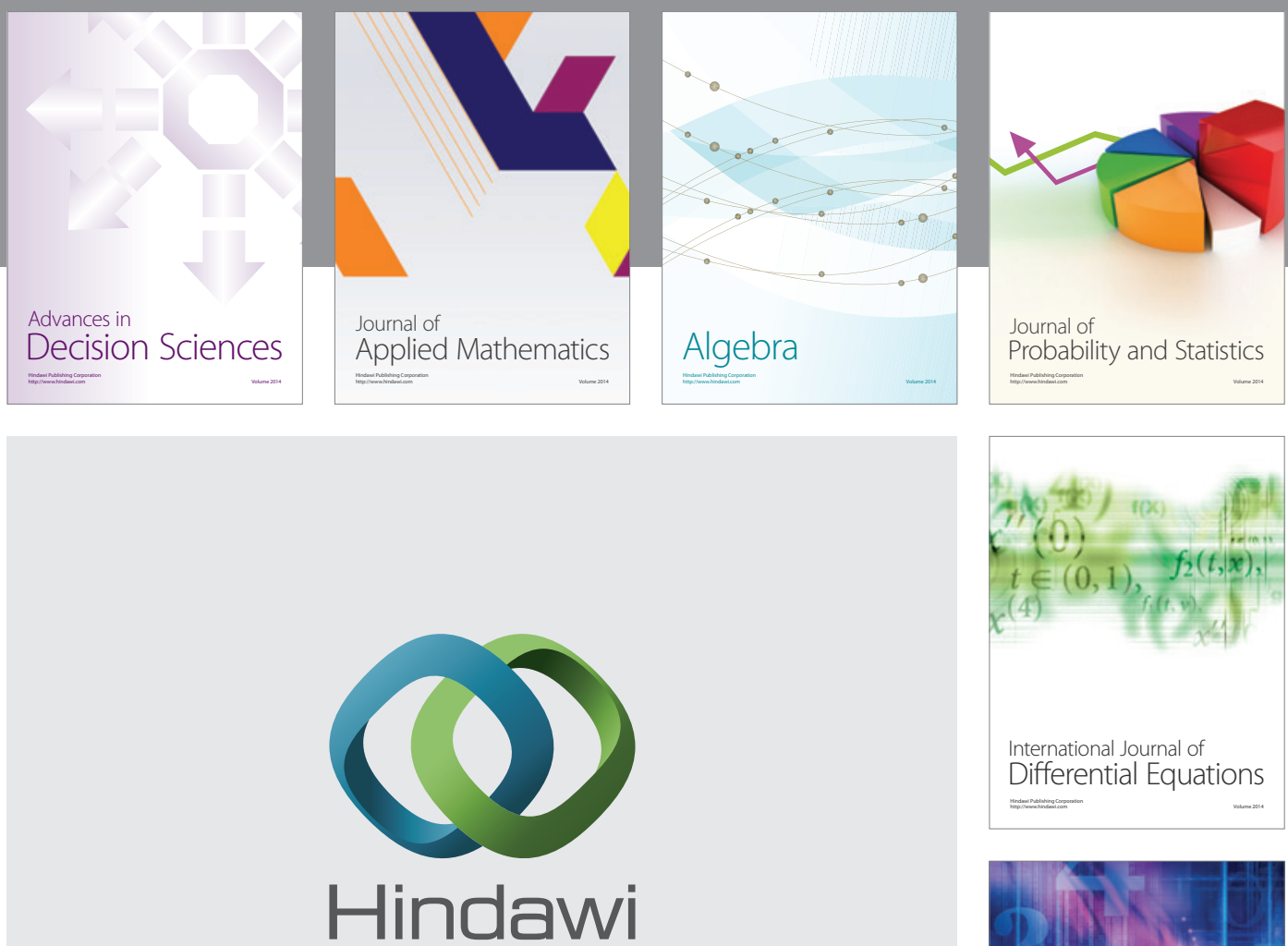

Submit your manuscripts at http://www.hindawi.com
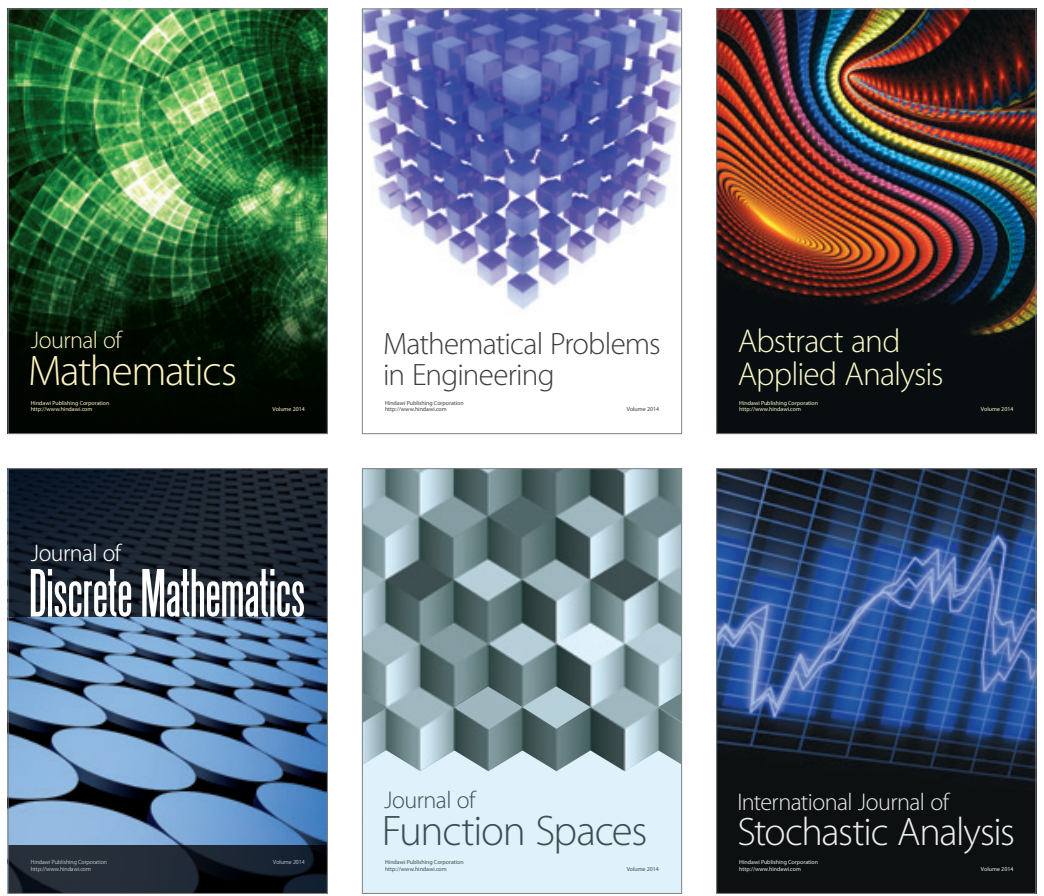

Journal of

Function Spaces

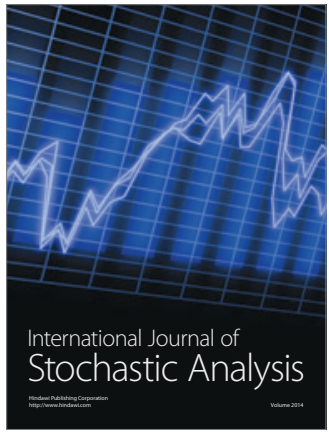

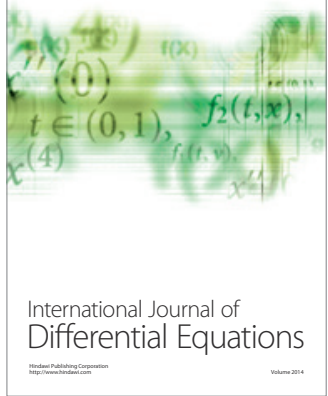
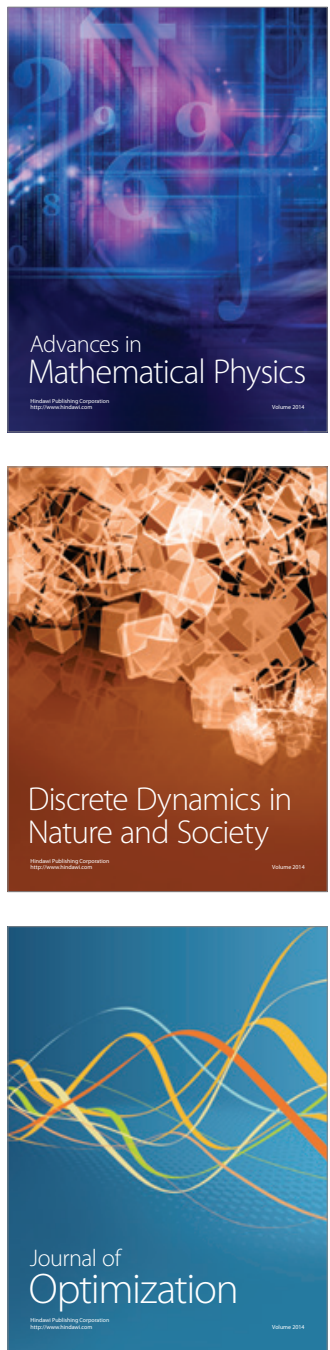